\title{
MAHALO Deep Cluster Survey I. Accelerated and enhanced galaxy formation in the densest regions of a protocluster at $z=2.5$
}

\author{
Rhythm Shimakawa, ${ }^{1 \star}$ Tadayuki Kodama, ${ }^{2}$ Masao Hayashi, ${ }^{3}$ J. Xavier Prochaska, ${ }^{1}$ \\ Ichi Tanaka, ${ }^{4}$ Zheng Cai, ${ }^{1}$ Tomoko L. Suzuki, ${ }^{3}$ Ken-ichi Tadaki ${ }^{3}$ and Yusei Koyama ${ }^{4}$ \\ ${ }^{1}$ UCO/Lick Observatory, University of California, 1156 High Street, Santa Cruz, CA 95064, USA \\ ${ }^{2}$ Astronomical Institute, Tohoku University, Aoba-ku, Sendai 980-8578, Japan \\ ${ }^{3}$ National Astronomical Observatory of Japan, Osawa, Mitaka, Tokyo 181-8588, Japan \\ ${ }^{4}$ Subaru Telescope, National Astronomical Observatory of Japan, National Institutes of Natural Sciences, 650 North A'ohoku Place, Hilo, HI 9672
}

Accepted XXX. Received YYY; in original form 2017 August 21

\begin{abstract}
We carried out deep $\mathrm{H} \alpha$ narrowband imaging with 10 hours net integrations towards the young protocluster, USS1558-003 at $z=2.53$ with the Subaru Telescope. This system is composed of four dense groups with massive local overdensities, traced by 107 $\mathrm{H} \alpha$ emitters (HAEs) with stellar masses and dust-corrected star formation rates down to $1 \times 10^{8} \mathrm{M}_{\odot}$ and $3 \mathrm{M}_{\odot} \mathrm{yr}^{-1}$, respectively. We have investigated the environmental dependence of various physical properties within the protocluster by comparing distributions of HAEs in higher and lower densities with a standard Kolmogorov-Smirnov test. At $97 \%$ confidence level, we find enhanced star formation across the star-forming main sequence of HAEs living in the most extreme 'supergroup', corresponding to the top quartile of overdensities. Furthermore, we derive distribution functions of $\mathrm{H} \alpha$ luminosity and stellar mass in group and intergroup regions, approximately corresponding to 30 times and 8 times higher densities than the general field. As a consequence, we identify 0.7 and 0.9 dex higher cutoffs in $\mathrm{H} \alpha$ luminosity and stellar mass functions in the dense groups, respectively. On the other hand, HAEs in the intergroup environment of the protocluster show similar distribution functions to those of field galaxies despite residing in significant overdensities. In the early phase of cluster formation, as inferred from our results, the densest parts in the protocluster have had an accelerated formation of massive galaxies. We expect that these eventually grow and transform into early-type galaxies at the bright end of the red sequence as seen in present-day rich clusters of galaxies.
\end{abstract}

Key words: galaxies: formation - galaxies: evolution - galaxies: high-redshift

\section{INTRODUCTION}

Galaxy protoclusters ${ }^{1}$ (van Albada 1961; Peebles 1970; Sunyaev \& Zeldovich 1972) provide us with direct insight into the early phase of hierarchical inside-out growth of the galaxy clusters. Studying these unique laboratories also leads deep understanding such as formation histories of strong colour-magnitude relationships as known as the red sequence (de Vaucouleurs 1961; Visvanathan \& Sandage 1977; Butcher \& Oemler 1984; Bower et al. 1992, 1998; Terlevich et al. 2001; Tanaka et al. 2005; Kodama et al. 2007; Mei et al. 2009) and physical origins of galaxy segregation

^ rhythm@ucolick.org i.e. morphology-density relation as seen in the local galaxy clusters (e.g. Dressler 1980; Dressler et al. 1997; Couch et al. 1998; Goto et al. 2003, and more modern examples by Cappellari et al. 2011; Houghton et al. 2013; Fogarty et al. 2014; Brough et al. 2017).

Protoclusters at redshifts greater than two have been extensively searched for and studied over the past two

\footnotetext{
1 Various survey bias and restrictions result in vague and inconsistent definitions of the protocluster in any work. This series of papers refers to overdense regions on the spatial scale of $\gtrsim 10$, $\sim 1-10$, and $\lesssim 1$ comoving Mpc as large-scale structures, protoclusters, and dense groups (or cores) for the target, respectively.
} 
decades (e.g. Steidel et al. 1998; Kurk et al. 2000, 2004; Venemans et al. 2002; Mayo et al. 2012; Shimakawa et al. 2014; Planck Collaboration et al. 2015; Toshikawa et al. 2016; Cai et al. 2016; Toshikawa et al. 2017). The results and conclusions from the past studies are not always consistent, but still, these do not have to be coherent with each other since they are based on different protoclusters with various overdensities, scale, sample selection, and redshifts (e.g. conflicts in gaseous metallicities of protocluster galaxies: Kulas et al. 2013; Shimakawa et al. 2015a; Valentino et al. 2015; Kacprzak et al. 2015; Tran et al. 2015). As a general tendency, however, one tends to confirm a larger number of massive and/or red star-forming galaxies in massive protoclusters as compared to the general field (Steidel et al. 2005; Hatch et al. 2011; Matsuda et al. 2011b; Hayashi et al. 2012; Koyama et al. 2013a,b; Wang et al. 2016; Husband et al. 2016; Hatch et al. 2017; Ao et al. 2017; Oteo et al. 2017). This implies that rapid and early build-up of bright red sequence objects has occurred in a young phase of protocluster formation. Indeed, massive clusters of galaxies at lower- $z$ host a higher fraction of very massive quiescent galaxies than lower-density environments, just like skyscrapers in the big city (Balogh et al. 2001; Bamford et al. 2009; Blanton \& Moustakas 2009; Muzzin et al. 2012; Darvish et al. 2016; Tomczak et al. 2017).

In the local Universe, Bamford et al. (2009) and Skibba et al. (2009) reported that, based on data from the Galaxy Zoo project (Lintott et al. 2008, 2011), the strong colour dependence remains prominent at fixed stellar mass in the sense that the high-density environments tend to have a higher fraction of red galaxies (see also Zehavi et al. 2011; Kelkar et al. 2017). This trend is particularly decisive at the lower mass regime, suggesting that local overdensities would control those quenching histories more efficiently (Peng et al. 2010b; Thomas et al. 2010; Bolzonella et al. 2010; Mortlock et al. 2015). More interestingly, however, the (kinematic) morphology-density relation is much less prominent when one compares galaxies across density environments for a given stellar mass range (Bamford et al. 2009; Brough et al. 2017). Bamford et al. (2009); Brough et al. (2017) argue that such morphological trends are primarily driven by stellar mass rather than environments, while the larger number of very massive galaxies in clusters of galaxies can give an apparent morphology-density relation in these systems.

Due to this background, high- $z$ protoclusters are ideal testbeds for understanding the physical processes that drive substantial excess of bright red sequence galaxies in today's cluster centres since these massive galaxies are expected to be built in the early stage of cluster formation at $z>2$ (e.g. Kodama et al. 2007; Zirm et al. 2008; Snyder et al. 2012; Strazzullo et al. 2016, and see also theoretical work by e.g. Romeo et al. 2005; Jiménez et al. 2011; Gabor \& Davé 2012; Behroozi et al. 2013). However, early environmental dependence of massive galaxy formation remains controversial based solely on the perspective of analyses of passive galaxies in clusters or protoclusters (Thomas et al. 2005, 2010; Strazzullo et al. 2013; Gobat et al. 2013; Tanaka et al. 2013; Newman et al. 2014; Ferré-Mateu et al. 2014). To seek a clearer picture, we require a more direct investigations towards the massive forming galaxies in the young protoclusters at high redshifts where star formation is still actively ongoing.
With this motivation, addressing the stellar mass function of forming galaxies in the early phase of protoclusters is essential to identify the physical origins of the environmental dependence of the stellar mass function for early type galaxies in today's galaxy clusters. Also, those star formation and its distribution provide us with original insight into the formation histories of massive red sequence galaxies. Toward these particular goals, we have carried out the MAHALO Deep Custer Survey (MDCS) as an extension of our previous systematic campaign the Mapping H-Alpha and Lines of Oxygen with Subaru (MAHALO-Subaru, Kodama et al. 2013). Both projects are designed to search and map $\mathrm{H} \alpha$ or [OII] line-emitting galaxies associated with distant clusters, protoclusters, and random fields at certain redshift slices traced by our narrowband filters. The narrowband filters are used with the Subaru Prime Focus Camera (Suprime-Cam; Miyazaki et al. 2002) or the Multi-Object InfraRed Camera and Spectrograph (MOIRCS; Ichikawa et al. 2006; Suzuki et al. 2008) on the Subaru Telescope. The MCDS aims to get deeper $\mathrm{H} \alpha$ imaging data for two dense protocluster regions among our parent sample, PKS 1138-262 at $z=2.2$ (Koyama et al. 2013a) and USS 1558-003 at $z=2.5$ (Hayashi et al. 2012) with net integrations amounting to a total of 5 hrs and $10 \mathrm{hrs}$, respectively, which are extended from exposure time of $3 \mathrm{hrs}$ for each by MAHALO-Subaru. The extended sample resolves spatial distributions of galaxies on the scale of $<1$ physical Mpc that resolves substructures within the protoclusters. High sampling density is quite essential for the study of such high- $z$ protoclusters since those structure formation would not be steady down yet, and spatial distributions of protocluster members are expected to be more scattered and clumpy (Muldrew et al. 2015; Chiang et al. 2017). Furthermore, a larger sample allows us to more easily determine the characteristic $\mathrm{H} \alpha$ luminosity $\left(L_{\mathrm{H} \alpha}^{*}\right)$ and stellar mass $\left(M_{\star}^{*}\right)$ on these distribution functions and may also provides insight into those faint-end profiles (i.e. the slope of power law, $\alpha$ ). These will help us to know if local environment can drive stellar mass assembly histories in an early phase of cluster formation at high redshift.

This work represents the full results obtained from the $10 \mathrm{hrs}$ deep narrowband imaging for USS 1558-003 at $z=2.5$ followed by our initial report by Hayashi et al. (2016). Our analyses improve sample selection $(\S 2)$. We then compare various physical properties and $\mathrm{H} \alpha$ luminosity and stellar mass functions of obtained sample across local overdensities $(\S 3)$. Section $\S 4$ describes discussions on similarities and differences between HAEs in higher and lower densities, and we summarise them in the final part $(\S 5)$. This paper assumes the cosmological parameters of $\Omega_{M}=0.3, \Omega_{\Lambda}=0.7$ and $h=0.7$ and employs a Chabrier (2003) stellar initial mass function. The AB magnitude system (Oke \& Gunn 1983) are used throughout the paper.

\section{TARGET AND DATASET}

\subsection{USS 1558-003}

USS $1558-003\left(\alpha_{\mathrm{J} 2000}=16^{\mathrm{h}} 01^{\mathrm{m}} 17^{\mathrm{s}}, \delta_{\mathrm{J} 2000}=-00^{\mathrm{d}} 28^{\mathrm{m}} 47^{\mathrm{s}}\right.$, hereafter USS 1558) is located at $z=2.53$, the highest redshift where the $\mathrm{H} \alpha$ emission line is observable from the ground. USS 1558 was first discovered as an overdensity by a 
factor of four in red galaxies associated with the radio galaxy (RG) USS 1558-003 (Kajisawa et al. 2006), but without a prominent red sequence (Kodama et al. 2007; Galametz et al. 2012). Instead, we see four overdense groups associated with $\mathrm{H} \alpha$-emitting galaxies (Hayashi et al. 2012, 2016). These suggest that this protocluster system is a young, unrelaxed phase in contrast to more advanced cluster systems at similar redshifts. Observationally, USS 1558 is a valuable target for studying young protocluster systems since this is at a redshift supported by $\mathrm{H} \alpha$ line and brighter than more distant protoclusters.

The density peak of USS 1558 corresponds to an approximately 30 times higher number density of $\mathrm{H} \alpha$-selected starforming galaxies relative to the general field (Hayashi et al. 2012). This object is the densest star-forming protocluster discovered to date at $z>2$ compared with other familiar massive protoclusters such as PKS 1138-262 (Kurk et al. 2000; Koyama et al. 2013a), 4C23.56 at $z=2.5$ (Knopp \& Chambers 1997; Tanaka et al. 2011), HS $1700+643$ at $z=2.3$ (Steidel et al. 2005; Erb et al. 2011), 2QZ at $z=2.3$ (Matsuda et al. 2011b; Kato et al. 2016), and Cl J1449+0856 at $z=2.0$ (Gobat et al. 2011, 2013). The overdensity is such that the mean projected separation of HAEs in the density peak is smaller than $100 \mathrm{kpc}(\S 3.1)$. Thus, it is highly likely that their sub-haloes begin to overlap one another common group halo.

Four dense groups of HAEs (Hayashi et al. 2016) align from the northwest to the southeast (NW-SE) direction: a small group located in the north of the RG, a loose group around the RG and six distant red galaxies without spectroscopic confirmation (Hayashi et al. 2012), the richest clump at 3.5 arcmin away from the RG containing 25 HAEs within just $1 \operatorname{arcmin}^{2}$, and a small clump in between the two. The spatial distribution is also available in Fig. 3 of this paper. These structures are well aligned along the line of sight according to existing spectroscopic data (Shimakawa et al. 2014). The dense groups are likely to eventually merge and become a single rich galaxy cluster system. Although we do not know whether these groups are virialised, the dynamical masses are estimated to be $\sim 1 \times 10^{14} \mathrm{M}_{\odot}$ for the densest group region when we assume local virialisation (Shimakawa et al. 2014) according to the Finn et al. (2005) prescription based on the measured velocity dispersion. Such a massive overdensity may grow into today's most massive clusters like Coma (Chiang et al. 2013).

\subsection{Data}

This work combines the multi-broadband and deep narrowband images (Fig. 1) taken from the early data of the MAHALO-Subaru campaign (S10B-028I, Kodama et al.; Hayashi et al. 2012; Kodama et al. 2013), the new data by MAHALO Deep Cluster Survey (MDCS: S15A-047, Kodama et al.; Hayashi et al. 2016), and deep F160W/WFC3 photometry taken by the Hubble Space Telescope (HST). Although the data are already described by Hayashi et al. (2016), we summarise the observational details for the recent deep follow-up narrowband and broadband imaging with MOIRCS on the Subaru Telescope through the MDCS program.

In this observing run, we mainly focused on narrowband imaging with $N B 2315\left(\lambda_{\text {center }}=2.317 \mu \mathrm{m}, \mathrm{FWHM}=260 \AA\right)$

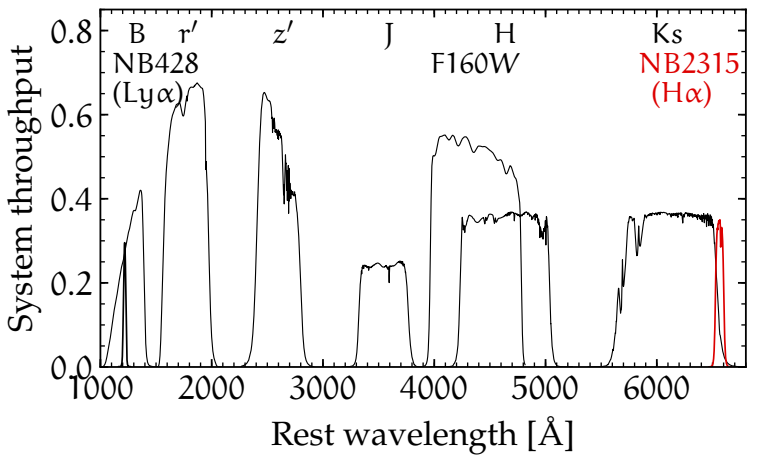

Figure 1. System throughputs of available photometric band data (Table 1) for the USS 1558 protocluster field. Rest wavelength is based on the protocluster redshift $z=2.53$. The $N B 2315$ filter has a companion filter, $N B 428$ for the Ly $\alpha$ line at the same redshift slice (see Shimakawa et al. 2017b,a). This paper uses NB428 photometry only to select secure HAE members showing both $\mathrm{H} \alpha$ and $\mathrm{Ly} \alpha$ flux excesses in the pair of narrowband filters (see text).

that can capture the $\mathrm{H} \alpha$ emission line from galaxies associated with USS 1558 at $z=2.53 \pm 0.02$. Observations were carried out during April 30 and May 6, 2015, under photometric conditions and with seeing of FWHM $\lesssim 0.6$ arcsec. The integration time of $380 \mathrm{~min}$ split to $180 \mathrm{sec}$ individual exposures. Furthermore, we took $J(116 \mathrm{~min})$ and $K_{S}$ (150 min) imaging. Combined with our previous data, this observing run has reconstructed those photometric data by total integration times of $191 \mathrm{~min}$ for $J, 207 \mathrm{~min}$ for $K_{S}$, and $583 \mathrm{~min}$ for $N B 2315$, respectively.

Reconstructed $J, K_{S}, N B 2315$ data were reduced very carefully by using the reduction pipeline MCSRED ${ }^{2}$ (Tanaka et al. 2011). MCSRED is composed of $\operatorname{IRAF}^{3}$ script. The reduction procedures include flat fielding, masking objects from the combined data in the 1st run (thus the whole reduction process was conducted twice to remake secure object masks), sky subtraction (by median sky and then a polynomiallyfitted surface for residual sky subtraction), distortion correction, and image mosaicing. Those processes can be executed semi-automatically. Throughout these processes, we eventually reconstructed deeper $J, K_{S}$, and $N B 2315$ data.

Taken together, $B, r^{\prime}, z^{\prime}, J, H, F 160 W, K_{S}$, and $N B 2315$ data are available in USS 1558. Those filter system throughputs are shown in Figure 1. Table 1 describes a brief overview of PSF-matched data where Galactic extinctions are derived from the NASA Extragalactic Database extinction law calculator ${ }^{4}$ which is based on the Fitzpatrick (1999) extinction law under an assumption of $R_{V}=3.1$ and Schlegel et al. (1998); Schlafly \& Finkbeiner (2011) for the dust reddening. We adopt $\mathrm{E}(\mathrm{B}-\mathrm{V})=0.1339$ mag by Schlafly \& Finkbeiner (2011).

\subsection{Sample selection}

This work is based on the narrowband emitter sample of Hayashi et al. (2016). Hayashi et al. (2016) have selected the

\footnotetext{
2 http://www.naoj.org/staff/ichi/MCSRED/mcsred.html

3 http://iraf.noao.edu

4 http://irsa.ipac. caltech.edu/applications/DUST/
} 
Table 1. Data summary (the same as Table 1 in Hayashi et al. 2016). The first to seventh columns indicate filter name, instrument/telescope, filter centre wavelength, and filter FWHM, $5 \sigma$ limiting magnitude in 1"2 aperture diameter, and galactic extinction, respectively. The eighth column shows the references for each data.

\begin{tabular}{lccccccc}
\hline Filter & Instrument/Telescope & $\begin{array}{c}\lambda_{\text {center }} \\
(\mu \mathrm{m})\end{array}$ & $\begin{array}{c}\text { Integration } \\
(\mathrm{min})\end{array}$ & $\begin{array}{c}\text { FWHM } \\
(\operatorname{arcsec})\end{array}$ & $\begin{array}{c}5 \sigma \\
(\mathrm{AB})\end{array}$ & $\begin{array}{c}\mathrm{A}_{\lambda} \\
(\mathrm{mag})\end{array}$ & Reference \\
\hline$N B 2315$ & MOIRCS/Subaru & 2.32 & 583 & 0.67 & 23.90 & 0.05 & Hayashi et al. $(2012,2016)$ \\
$K_{S}$ & MOIRCS/Subaru & 2.15 & 207 & 0.67 & 24.49 & 0.05 & Hayashi et al. $(2012,2016)$ \\
$H$ & MOIRCS/Subaru & 1.64 & 45 & 0.67 & 23.78 & 0.07 & Hayashi et al. $(2012,2016)$ \\
$F 160 W$ & WFC3/HST & 1.54 & 87 & 0.21 & 27.46 & 0.08 & Hayashi et al. $(2012,2016)$ \\
$\boldsymbol{J}$ & MOIRCS/Subaru & 1.25 & 191 & 0.67 & 24.85 & 0.11 & Hayashi et al. (2012, 2016) \\
$z^{\prime}$ & Suprime-Cam/Subaru & 0.91 & 55 & 0.67 & 26.03 & 0.19 & Hayashi et al. $(2012)$ \\
$r^{\prime}$ & Suprime-Cam/Subaru & 0.63 & 90 & 0.67 & 27.24 & 0.35 & Hayashi et al. $(2012)$ \\
$B$ & Suprime-Cam/Subaru & 0.45 & 80 & 0.70 & 27.51 & 0.55 & Hayashi et al. $(2012)$ \\
\hline
\end{tabular}

sample as these sources showing more than $3 \sigma$ flux excess at $N B 2315$ relative to $K_{S}$ band photometry in the following criteria,

$$
\begin{aligned}
K s-N B & >-2.5 \log \left(1-\frac{\sqrt{f_{3 \sigma, K s}+f_{3 \sigma, N B}}}{f_{N B}}\right)+0.1 \\
K s-N B & >0.35
\end{aligned}
$$

where $f_{N B}, f_{3 \sigma, K s}$, and $f_{3 \sigma, N B}$ are narrowband flux density, $3 \sigma$ limit of $K_{S}$-band flux density, and $3 \sigma$ limit of narrowband flux density, respectively. We assumed two sigma limiting magnitude in $K_{S}$-band for faint $K_{S}$ sources, which amount to 26 objects in 177 selected narrowband emitters (Hayashi et al. 2016). The $3 \sigma$ flux limit is $1 \times 10^{-17} \mathrm{erg} \mathrm{s}^{-1} \mathrm{~cm}^{-2}$, corresponding to a SFR limit of $2.4 \mathrm{M}_{\odot} \mathrm{yr}^{-1}$ for $z=2.53$ assuming the the Kennicutt (1998) prescription. The latter colour cut corresponds to the $\mathrm{H} \alpha$ line equivalent width (EW) limit of $45 \AA$ in the rest frame at $z=2.53$. One should note that the NB2315 filter is located at the red end of $K_{S}$-band, which requires a colour-term correction for $\mathrm{H} \alpha$ line flux and $\mathrm{EW}$ measurements. This may also lead us to overestimate $\mathrm{H} \alpha$ fluxes in redder objects. We carefully checked and corrected this effect ( $\$ 3.2$ and Appendix B) and find that it should have a negligible impact on our results and conclusions.

A subset of our HAE sample will be emitters of other emission lines in the foreground and background. According to Sobral et al. (2013) and An et al. (2014), $\mathrm{H} \alpha$ and [OIII] emitters would consist mostly of K-band selected emitters, which account for approximately $40-50$ and 20-30 per cents of the entire emitter sample in the general fields, respectively. To restrict the sample to a more likely set of protocluster members, we need to conduct further analyses. We first selected secure protocluster members which were confirmed by previous follow-up spectroscopy (Shimakawa et al. 2014,2015 b) or Ly $\alpha$ narrowband imaging (Shimakawa et al. 2017a). These confirmed HAEs amount to a total of 49 objects (Table 2). Hayashi et al. (2016) carried out further colour-colour selection i.e. $r^{\prime} J K s$ and $r^{\prime} H_{F 160 W} K_{S}$ to select HAEs at $z=2.53 \pm 0.02$ for narrowband emitters without such identifications. We revisit these colour selection criteria to select protocluster members more effectively, and finally revise them by new rules on $r^{\prime} J K_{S}$ and $B r^{\prime} K_{S}$ colourcolour selections with the help of spectroscopically confirmed members and spec- $z$ sources by MOSDEF survey (the MOSFIRE Deep Evolution Field survey, second redshift release by Kriek et al. 2015). In the latter sample, we employ only galaxies in the COSMOS-CANDELS field, which allow us to use the same broadband datasets as for USS 1558 based on the 3D-HST database (Brammer et al. 2012; Skelton et al. 2014). The colour-colour selections are shown in Figure 2, and the selection criteria are defined as follows,

$$
\begin{array}{lll}
r^{\prime}-J<0.9 & \text { or } & (J-K s)>\left(r^{\prime}-J\right)-0.6 \\
B-r^{\prime}<1.0 & \text { or } & \left(r^{\prime}-K s\right)>2.5 \times\left(B-r^{\prime}\right)-1.6 .
\end{array}
$$

$r^{\prime} J K_{S}$ can be used to remove low- $z$ contaminants at $z<1.6$ as demonstrated in $B z^{\prime} K_{S}$ selections (Daddi et al. 2004; Sobral et al. 2013). We here use this selection to mainly remove [SIII] $\lambda \lambda 9071,9533$ emitters at $z=1.4-1.6$ which may be one of the major contaminations according to An et al. (2014). Then, $B r^{\prime} K_{S}$ colour cut is useful to reject background [OIII] $\lambda \lambda 4960,5008$ emitters at $z \sim$ 3.6. Since those Lyman break features stride across between $B$ and $r^{\prime}$-band wavelengths, their $B-r^{\prime}$ colours are distinctly redder than those of galaxies at $z \sim 2$. In this colour selection, our sample at $z=2.5$ tends to have redder $B-r^{\prime}$ colours than the MOSDEF sources at the typical spectroscopic redshift of $z=2.3$ because those of our HAEs are closer to the Lyman break.

These colour criteria allow us to select an additional 58 HAEs and remove a total of 19 narrowband emitters from the entire sample (Table 2). We assume $2 \sigma$ limiting magnitudes for non-detections, and then classify them if their upper limits or lower limits satisfy the colour criteria. Combined with 49 confirmed members (Shimakawa et al. 2014, 2015b, 2017a), we adopt 107 narrowband emitters as the HAE protocluster sample. It should be noted that those colour criteria are not guaranteed to select HAEs or to remove foreground and background emitters. In particular, part of colour-selected HAEs and rejected contaminants are located near boundaries of the criteria, and we cannot securely classify them taking account of those photometric errors. Further spectroscopic observation is needed to reliably identify whether or not they are protocluster members. Lastly, there are 51 unclassified emitters that cannot be classified by the colour criteria due to the non-detection in at least one band. These are defined as HAE candidates. Given that our target is the dense protocluster region, the majority of such faint emitters should be HAEs. The contamination rate of HAE candidates can be roughly expected to be $\sim 15$ per cent given 19 rejected objects among $126(107+19)$ brighter emitters. 

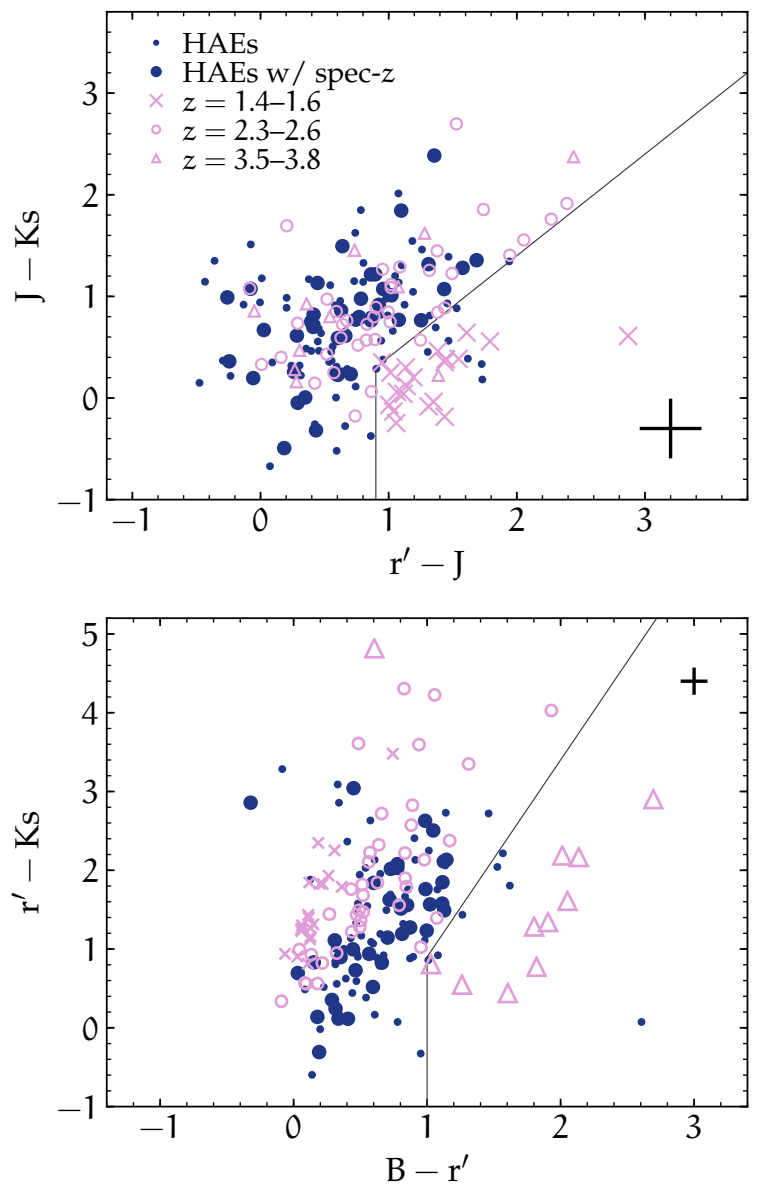

Figure 2. $r^{\prime} J K_{S}$ (upper panel) and $B r^{\prime} K_{S}$ (lower panel) colourcolour selections for USS 1558. Large and small blue filled circles indicate the narrowband emitters with and without confirmation by spectroscopy or a high Ly $\alpha$ EW, respectively. Open pink triangles, circles, and crosses are the spec- $z$ samples at $z=3.0-3.3$. $z=2.3-2.6$, and $z=1.2-1.4$ from the MOSDEF survey (Kriek et al. 2015), respectively. Black lines are colour thresholds defined to remove those foreground or background contaminants. The black crosses on each panel show typical $1 \sigma$ errors of our narrowband emitters. One should note that only galaxies with better than $2 \sigma$ detections in all bands appear in these panels.

Table 2. Classification of narrowband emitters in USS1558-003. This work employs confirmed and colour-selected emitters as the HAE sample, which account for 85 per cent of the bright narrowband emitters that can be classified by our colour selection criteria. We also use HAE candidates in some cases.

\begin{tabular}{lrl}
\hline Class & N & Description \\
\hline NBEs & 177 & NB emitters (Hayashi et al. 2016) \\
HAEs (confirmed) & 49 & spec- $z$ or Ly $\alpha$ confirmation \\
HAEs (by colours) & 58 & selected by r'JKs and Br'Ks \\
other line emitters & 19 & rejected by r'JKs or Br'Ks \\
\hline Total HAEs & 107 & confirmed + colour-selected \\
HAE candidates & 51 & faint and ambiguous \\
\hline
\end{tabular}

\section{RESULTS}

Using the constructed HAE database of the protocluster USS 1558, this work compares various physical properties of HAEs between higher and lower densities within the young protocluster at $z=2.53$. This provides us with insight into the environmental dependence on galaxy formation in an early stage of inside-out evolution of the galaxy cluster. Our deep $\mathrm{H} \alpha$ imaging data enable an investigation of the environmental dependence of galaxy characteristics across a diversity of substructures in the protocluster on smaller than one physical Mpc (ph-Mpc) scale.

\subsection{Spatial distributions}

Spatial distributions of narrowband-selected line emitters can identify structures and substructures in and around the forming protoclusters, as demonstrated by the past studies (Kurk et al. 2004; Tanaka et al. 2011; Matsuda et al. 2011a; Hayashi et al. 2012; Koyama et al. 2013a; Cooke et al. 2014). We caution, however, that Ly $\alpha$ emitters which can be significantly missing in local overdensities (Shimakawa et al. 2017a). Furthermore, our narrowband imaging cannot trace low EW ( $<45 \AA$ ) and non $\mathrm{H} \alpha$-emitting (i.e. passive) galaxies. This should cause errors of density estimation which we cannot resolve as it stands. However, we stress that we would not miss significantly overdense substructures by HAEs since HAEs are well tracing the structure of distant red galaxies $\left(J-K_{S}>1.38\right.$; Franx et al. 2003) in this protocluster region (Hayashi et al. 2012).

To quantify environment, we define a density parameter, the mean projected distance $\bar{b}_{5 \text { th }}$, as described in the following manners:

$\bar{b}_{5 \text { th }}=2 \sqrt{\frac{1}{\pi \Sigma_{\mathrm{Nth}}}}$,

where $N$ is the number of HAEs within a radius of $r_{\mathrm{Nth}}$ that is the distance to the $(N-1)$ th neighbour from each HAE. $\Sigma_{\mathrm{Nth}}$ is the number density within the Nth radius $\left(=N / \pi r_{\mathrm{Nth}}^{2}\right)$. This paper employs $N=5$, and we note that the measured density parameters maintain relative consistencies even if we choose different $N$ values in the single-digit range. $\bar{b}_{5 \text { th }}$ expresses how close galaxies are to each other in projected space at $z \approx 2.53$ in the sense of lower values indicating higher densities. Also, this value can be regard as the familiar $\Sigma_{5 \text { th }}$ as seen in the equation 5 . The major reason to use $\bar{b}_{5 \text { th }}$ is that the mean projected distance can be used as a mean impact parameter which has been commonly employed in the Ly $\alpha$ absorption analyses (e.g. Wolfe et al. 2005; Fumagalli et al. 2010; Rakic et al. 2012). Since our forthcoming paper will discuss environmental effects on Ly $\alpha$ photon escape fraction as preliminary reported in Shimakawa et al. (2017a), the mean projected distance is an ideal indicator to quantify the local overdensities.

The spatial distribution and density structure of HAEs in USS 1558 are given by Figure 3. As reported by Hayashi et al. (2016), our MDC campaign succeeds in increasing the protocluster sample by 1.6 times as compared to the past survey (Hayashi et al. 2012). In our sample, 39 out of 107 HAEs are newly discovered as protocluster members and we also have additional 51 candidates (Table 2). A typical mean projected separation of $\bar{b}_{5 \text { th }}=220$ ph-kpc indicates 


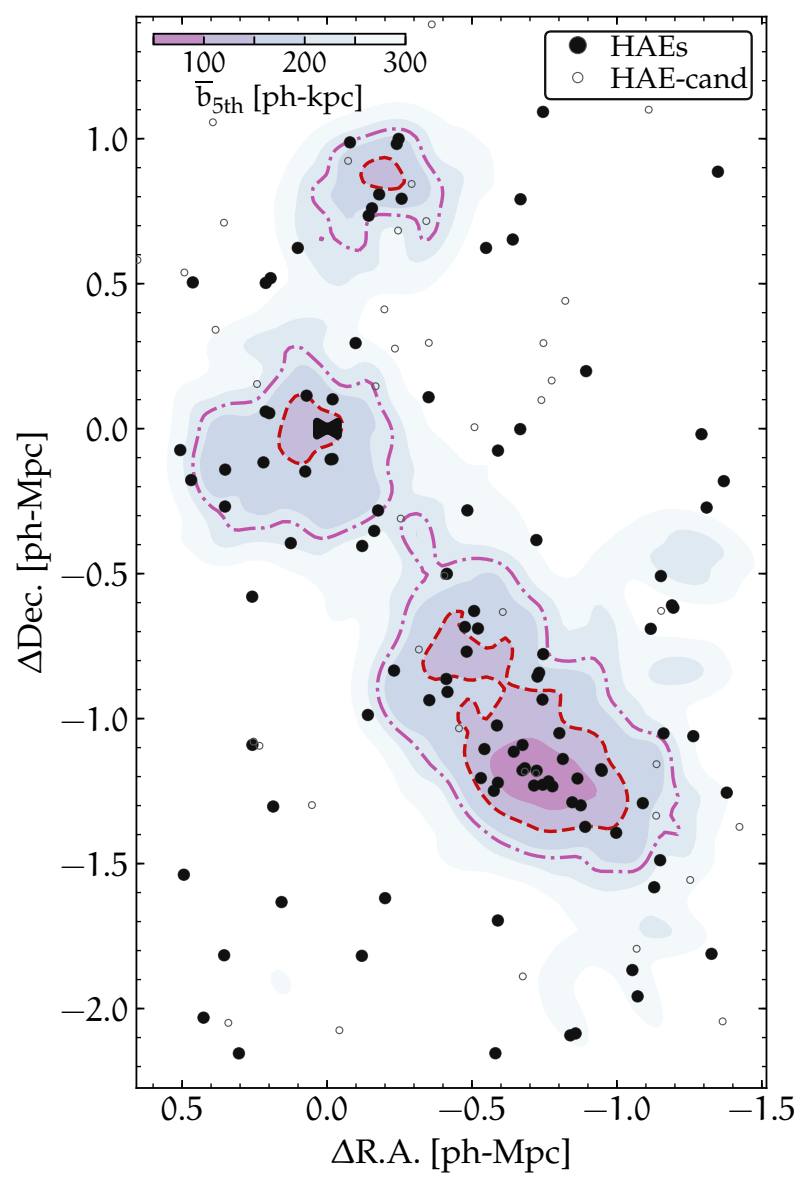

Figure 3. Spatial distribution of HAEs (filled circles) and HAE candidates (open circles). The bow-tie is the radio galaxy, USS 1558-003. Filled contours show the 5th mean projected separations of $\bar{b}_{5 \text { th }}<100,100-150,150-200,200-250,250-300$ phkpc, respectively. The contour map is smoothed by a 2D Gaussian kernel with $\sigma=1.5$ arcmin. The purple dash-dot contour line divides the sample into group and intergroup regions $\left(\bar{b}_{5 \text { th }}=220\right.$ ph-kpc or 50th percentile line). We also identify them at the top quartile high-density region as supergroup HAEs with the mean projected separation lower than $\bar{b}_{5 \text { th }}=150$ ph-kpc as marked by the red dashed curve.

that our target is associated with remarkably high overdensities, roughly corresponding to $15-20$ higher number density than the general field. It reaches to $\bar{b}_{5 \text { th }}<150 \mathrm{ph}-\mathrm{kpc}$ in the top quartile of high-density regions, which is comparable in size to virial radii of typical HAEs with a few times $10^{12}$ $\mathrm{M}_{\odot}$ halo masses (Cochrane et al. 2017). Such a heavy congestion implies that those haloes would overlap with each other sometimes physically and more easily along the line of sight, and also they might be sharing large common haloes with their neighbours.

To examine the effect of dependence within the protocluster, we divide the entire HAE sample into the group and intergroup samples by the median $\bar{b}_{5 \text { th }}$ value of $220 \mathrm{ph}-\mathrm{kpc}$ (dash-dot curve in Fig. 3). This criterion also corresponds to the $1 \sigma$ local overdensity in the survey area. Furthermore, we define a supergroup HAE sample to investigate environmental effects on some physical properties in the extraordinarily high density regions that reach $\bar{b}_{5 \text { th }}<150$ ph-kpc, which corresponds to the top quartile of overdensities in the protocluster (dashed curve in Fig. 3). While our deep data should enable the finest structure mapping for such a high- $z$ protocluster ever, one should note that the classification of the sample is data-driven rather than scientifically justified. We have no clear answer yet to properly evaluate substructures in high- $z$ protocluster for such $\mathrm{H} \alpha$-selected sample in the restricted area. Thus, this work simply compares galaxy properties between higher and lower densities in the same protoclustrer region, and leaves this issue for future study. Hi tomographic mapping by deep far-UV spectroscopic analyses (Lee et al. 2014, 2016) could be another, more direct solution to identify the substructures, although it is very difficult with current facilities to reach the spatial resolution of $\sim 1$ comoving Mpc (hereafter co-Mpc) necessary to resolve the protocluster halo by this kind of survey.

\subsection{Star-forming main sequence}

This section firstly repeats the derivation of stellar masses and SFRs previously done by Hayashi et al. (2016), but for the updated HAE samples. We then compare those between the group and intergroup environments. This work revises previous estimates of these parameters as described in the following sentences, implying slight systematic differences from the previous research. However, we stress that these revisions do not affect our conclusions.

We obtained the narrowband line flux $\left(F_{\mathrm{NB}}\right)$ and rest EW by the following formula,

$$
\begin{aligned}
F_{N B} & =\Delta_{N B}\left(f_{N B}-f_{K s^{\prime}}\right) \\
\mathrm{EW}_{N B, \text { rest }} & =\frac{F_{N B}}{f_{K s^{\prime}}}(1+z)^{-1}
\end{aligned}
$$

where $\Delta_{N B}$ is full-width-half-maximum (FWHM) of $N B 2315$ filter (260 $\AA$ ). $f_{K s^{\prime}}$ is the $K_{S}$-band flux density including the fixed colour term correction (0.07 mag) derived from modelinferred SEDs of HAEs (Fig. B2). We checked the impacts of this assumption by comparing measured line fluxes with those including the colour term correction in which we employ SED-inferred continua at the narrowband wavelength instead of $f_{K s^{\prime}}$. We find that the effect of the colour term variation on individual line fluxes should be negligible because they are consistent with each other within the standard dispersion of 0.065 dex. Indeed, our HAEs have high $\mathrm{EW}_{\mathrm{H} \alpha}$ (the median value of $180 \AA$ in the rest frame), and thus, line flux is dominant at the narrowband filter wavelength and the colour-term variation does not have a large effect such that similar studies caution for line emitters at lower redshifts (e.g. Vilella-Rojo et al. 2015). Thus, we stress that such a small uncertainty does not affect our results and conclusions.

After that, we carry out SED-fitting based on the SEDfitting code FAST distributed by Kriek et al. (2009). We used $B, r^{\prime}, z^{\prime}, J, H, F 160 W, K_{S}$ band photometry for the SED fitting. We should note that FAST code does not include nebular emission line components. This may cause systematic errors of stellar mass measurements of our sample, especially for lower-mass HAEs. Atek et al. (2011) reported that SED-inferred stellar masses for high EW (> $200 \AA$ ) galaxies without removing emission lines tend to be overestimated by a factor of two on average (see also e.g. Wuyts et al. 2007; Schaerer et al. 2013; Stark et al. 2013; Salmon et al. 2015). We believe that such a systematic error should be 


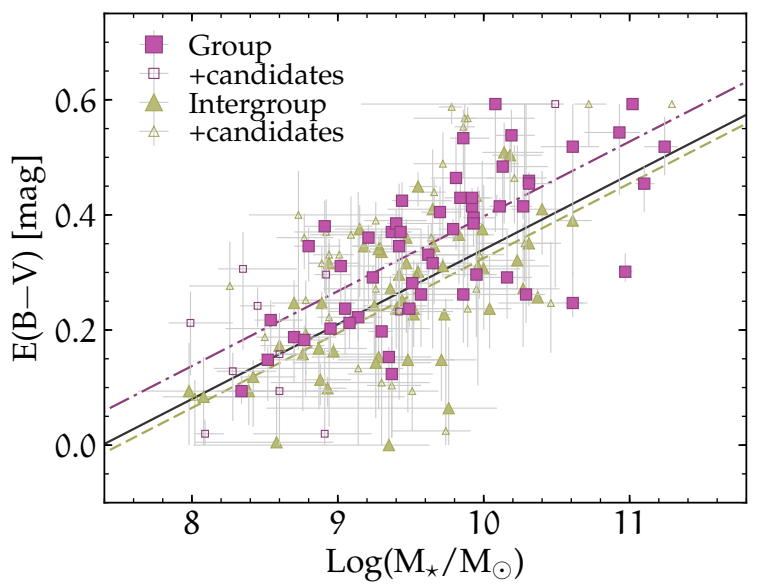

Figure 4. Stellar mass versus dust reddening, E(B-V) for HAEs (filled) and HAE (open) candidates. Purple squares and yellow triangles show the HAE samples in the group and intergroup regions, respectively. Solid, dash-dot, and dashed lines indicate the best fit line for the entire HAEs, group HAEs, and intergroup HAEs, respectively, where the latter two regressions assume the same slope as that for the entire HAEs. Errorbars are $1 \sigma$ errors based on the FAST measurements.

much smaller in our case since our deep $F 160 \mathrm{~W}(5 \sigma=27.5$ $\mathrm{mag})$ and $K_{S}(5 \sigma=24.5 \mathrm{mag})$ data that are critical for stellar mass estimations, are not affected by strong $\mathrm{H} \alpha$, [OIII], $\mathrm{H} \beta$ emission lines. SED fitting was conducted assuming a fixed redshift of $z=2.53$, based on the stellar population model of Bruzual \& Charlot (2003), the Calzetti et al. (2000) extinction curve, the Chabrier (2003) IMF, and fixed metal abundance of $Z=0.004\left(0.2 Z_{\odot}\right)$. We assume delayed exponentially declining star formation histories $(\mathrm{SFR} \propto t \exp (-t / \tau)$ ) where $\tau$ is allowed to be between $10^{9}$ and $10^{11}$ yr. Population ages between $10^{7.6}$ and $10^{9.4}$, and stellar extinction $\left(A_{V}\right)$ between 0 and $2.4 \mathrm{mag}$ are given, respectively. These values do not significantly affect stellar mass estimations, but the measurements of dust extinction systematically depend on input parameters. For example, measured dust extinctions $\left(A_{V}\right)$ in 80 per cent of HAEs change within a range of \pm 20 $\%$ uncertainties (and residuals vary even more) if we apply free parameters for $\tau$ and age given by the entire library. We ignore such a model dependency through the paper because this work solely focuses on the relative comparison of HAE samples selected in the same way, and based on the same datasets. $1 \sigma$ errors of obtained physical parameters are estimated by 100 Monte Carlo simulations attached with the FAST code.

The derived stellar masses and dust reddening are presented in Figure 4. The data are divided into group and intergroup samples by colour. As many of researchers have previously reported (e.g. Garn \& Best 2010; Reddy et al. 2010; Sobral et al. 2012; Ibar et al. 2013; Zahid et al. 2013; Whitaker et al. 2014; Koyama et al. 2015), these two parameters correlate with each other. The environmental dependence of measured stellar masses and dust extinctions of HAEs is discussed in $\S 3.4$ and $\S 3.5$.

We estimate $\mathrm{SFR}_{\mathrm{H} \alpha}$ of HAEs from the $\mathrm{H} \alpha$ narrowband fluxes based on the Kennicutt (1998) prescription assuming the Chabrier (2003) IMF (a factor of 1.7 reduction in SFR from the standard Kennicutt 1998 calibration). We cor- rect for $[\mathrm{NII}] \lambda \lambda 6550,6585$ line contamination to the narrowband flux by using the stellar mass- $[\mathrm{NII}] / \mathrm{H} \alpha$ relation for star-forming galaxies at a similar redshift (equation 20 in Steidel et al. 2014). Although Steidel et al. (2014) have selected the sample based on UV, sampling effect would be negligible since their mass-metallicity relation is consistent with that of $\mathrm{H}$-band selected galaxies at similar redshift (Sanders et al. 2015). In light of the small scatter in the mass-metallicity relation ( $0.1 \mathrm{dex})$, the typical error in [NII] flux can be estimated to be $<12$ per cent. We also calculate a dust extinction correction for the $\mathrm{H} \alpha$ emission line based on the SED-inferred stellar extinction $\left(A_{V}\right)$ by FAST and the Calzetti et al. (2000) extinction law under the assumption that $\mathrm{E}(\mathrm{B}-\mathrm{V})_{\text {stellar }}=\mathrm{E}(\mathrm{B}-\mathrm{V})_{\text {nebular }}$. Although we have to rely on this assumption because we have no $\mathrm{H} \beta$ line data for the Balmer decrement, this hypothesis is relatively reasonable at high redshifts (Reddy et al. 2015). One should note that ratio of nebular extinction to stellar extinction likely depends on galaxy properties, especially on SFR (Price et al. 2014; Reddy et al. 2015). This may mean that our SFR measurement may underestimate mainly the SFR of active and dusty star-forming objects which are expected at the massive end. However, it is challenging to estimate proper SFR values of such dusty starbursts in the rest optical regime in any case (Dannerbauer et al. 2014; Tadaki et al. 2017). More importantly, such massive and/or dusty HAEs are preferentially located in higher densities ( $\$ 3.4$ and $3.5)$, and thus, our comparison results and conclusions ( $\$ 5)$ will be enhanced once we could correct those nebular extinctions more precisely. Measured parameters of individual HAEs are summarised in Appendix A (Table A).

Figure 5 shows the dust-corrected SFRs of the HAEs as a function of stellar mass. Our HAE sample establishes a mass-SFR relation known as the star-forming main sequence, which is discovered by Brinchmann et al. (2004) in the local Universe and also by Daddi et al. (2007); Elbaz et al. (2007); Noeske et al. (2007) at high redshifts, and then followed and improved by so many studies (e.g. Whitaker et al. 2012; Speagle et al. 2014; Oteo et al. 2015; Shivaei et al. 2015; Tomczak et al. 2016). The SFR limit of our narrowband observations is 2.4 or $6.0 \mathrm{M}_{\odot} \mathrm{yr}^{-1}$ with $A_{\mathrm{H} \alpha}=0$ or 1 mag, respectively. Because of this SFR limit, our sample is biased toward highly active HAEs in the low-mass regime especially below $\mathrm{M}_{\star} \lesssim 10^{9.4} \mathrm{M}_{\odot}$. In other words, our HAE sample is relatively complete above this stellar mass range, although low line EW $(<45 \AA)$ galaxies and heavilyobscured HAEs will be absent.

Figure 5 shows that our HAE sample well follows the star-forming main sequence reported by Oteo et al. (2015) for $\mathrm{H} \alpha$-selected galaxies in the general field at $z=2.2$. The typical offset value from the main sequence of Oteo et al. (2015) is estimated to be 0.15 dex. This small offset may be caused by redshift evolution $(z=2.2$ vs. $z=2.5)$, environmentally-driven effects, or different extinction corrections. Besides, our deep $\mathrm{H} \alpha$ imaging goes down to by 2.4 times fainter $\mathrm{H} \alpha$ luminosities than the previous data (Hayashi et al. 2012), which enables discovery of 20 active low-mass HAEs $\left(\mathrm{M}_{\star}<10^{9} \mathrm{M}_{\odot}\right)$ with very high specific SFRs (sSFRs $)>(0.1 \mathrm{Gyr})^{-1}$. This paper does not touch the detail of such unique emitters since those are already discussed by Hayashi et al. (2016).

The right panel in the Fig. 5 represents the same as the 

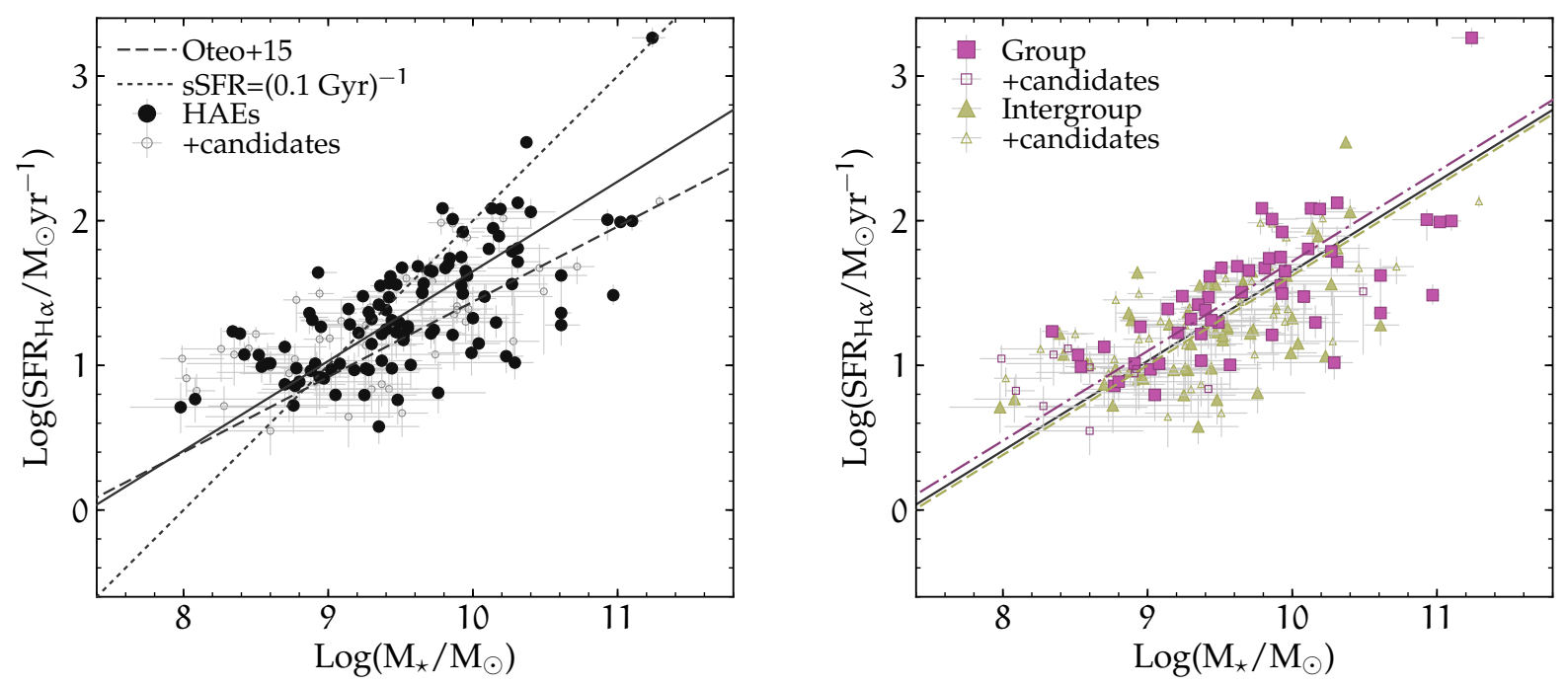

Figure 5. Stellar mass versus SFR from dust-corrected $\mathrm{H} \alpha$ luminosity. Left and right panel show the same results, but the right figure changes the colours of HAEs in group (purple squares) or intergroup (yellow triangles) region. Filled and open symbols indicate HAEs and HAE candidates (Table 2), respectively. The black solid and dashed line show $\mathrm{M}_{\star}-\mathrm{SFR}$ relations for our HAE sample and HAEs in the general field at $z=2.2$ (Oteo et al. 2015), respectively. Oteo et al. (2015) have corrected dust extinction with a mass-dependent prescription (Garn \& Best 2010). Purple dash-dot and yellow dashed lines are the median-fitted lines for group and intergroup HAEs, assuming the same slope $\left(\mathrm{SFR} \propto \mathrm{M}_{\star}^{0.62}\right)$ as that for the entire HAEs. The black dotted line indicates $\mathrm{sSFR}=(0.1 \mathrm{Gyr})^{-1}$.

left, but here we separate the sample into HAEs in group $\left(\bar{b}_{5 \text { th }}<220\right.$ ph-kpc) and intergroup $\left(\bar{b}_{5 \text { th }}>220\right.$ ph-kpc $)$ environments as classified in the previous section. The median SFR of HAEs in group and intergroup regions are $30 \mathrm{M}_{\odot} \mathrm{yr}^{-1}$ and $17 \mathrm{M}_{\odot} \mathrm{yr}^{-1}$, respectively, and the Kolmogorov-Smirnov (KS) test suggests the dense group environments tend to have more active HAEs by the $p$-value of 0.005 . For a given stellar mass, however, those two populations are no longer statistically different $(p=0.353)$ as inferred from the comparison of the offset from the median-fitted main sequence (hereafter $\Delta \mathrm{MS}$ ) in the stellar mass range of $10^{9-10.5} \mathrm{M}_{\odot}$. This means that HAEs shift upper rightward approximately along the main-sequence depending on the local overdensity. Such trends broadly agree with Koyama et al. (2013a,b) that have reported as up-rightward shift along the main sequence in the protocluster at $z=2.2$ as compared to the field at the same redshift. The section $\S 3.5$ summarises the results of the KS test for physical parameters.

Surprisingly, however, we identify even clearer SFR differences across local densities when we compare those between the supergroup and the intergroup (Fig. 6). The KS test indicates those two populations are discriminable from each other $(p=0.03)$ even if we compare those for a given stellar mass i.e. $\triangle$ MS. $81 \%$ of the supergroup HAEs consist of the densest star-forming clump in SW area in the protocluster (Fig. 3) where the mean projected separation reaches $\bar{b}_{5 \text { th }}<150$ ph-kpc or $<640$ ph-kpc in the 3 -D space. In the supergroup region, HAEs with $\mathrm{M}_{\star}<10^{10.4} \mathrm{M}_{\odot}$ are located at the upper envelope of the main sequence of HAEs in the intergroup regions, suggesting that extremely high densities accelerate galaxy formation. Seven out of 26 HAEs in the supergroup region have projected distances of $<30$ ph-kpc to the nearest neighbours, and thus, some of those may be merger-induced starburst galaxies.

One critical concern for clear SFR enhancement in the supergroup regions is the uncertainties of extinction correc- tions for individual HAEs. However, we find a significant excess even without dust corrections $(p=0.03)$. Another issue is a systematic error due to the filter response function of the NB2315 narrowband filter (that is not a perfect top-hat function). We run another simulation to test this possibility, and then find that a quite drastic situation should be required to explain the difference only by the error of filter flux loss (see Appendix C2). Thus, enhanced star formation of HAEs in the extremely dense group regions is more likely an intrinsic phenomenon. That said, deep follow-up NIR spectroscopy is highly desired to confirm SFR enhancement in the supergroup regions.

The standard deviation of the main sequence is also worth investigating (Lin et al. 2012; Koyama et al. 2014), for instance, to test if dense environments enhance the merger rate and/or then increase the number of starburst galaxies (Gottlöber et al. 2001; Genel et al. 2014), and drive environmental quenching (Wetzel et al. 2013; Gobat et al. 2015). However, the observed scatter of the star-forming main sequence show consistent values of 0.33 and 0.34 dex between group and intergroup regions, which also agree with typical value of $\sim 0.3 \mathrm{dex}$ in the general field at the similar redshifts reported by past studies (e.g. Speagle et al. 2014; Shivaei et al. 2015). More interestingly, HAEs at $M_{\star}<10^{10.4} M_{\odot}$ in the densest regions $\left(\bar{b}_{5 \text { th }}<150\right.$ ph-kpc) have a tighter main sequence $(\sigma=0.19$ dex or 1.5 times smaller than that of intergroup HAEs on a linear scale). We should note, however, that our $\mathrm{H} \alpha$ survey may miss dust-hidden starbursts and we may underestimate dusty HAEs in our sample. Also, our narrowband survey cannot detect objects with declining star formation with low $\mathrm{H} \alpha$ EWs. These uncertainties may lead us to underestimate the main sequence scatter. 


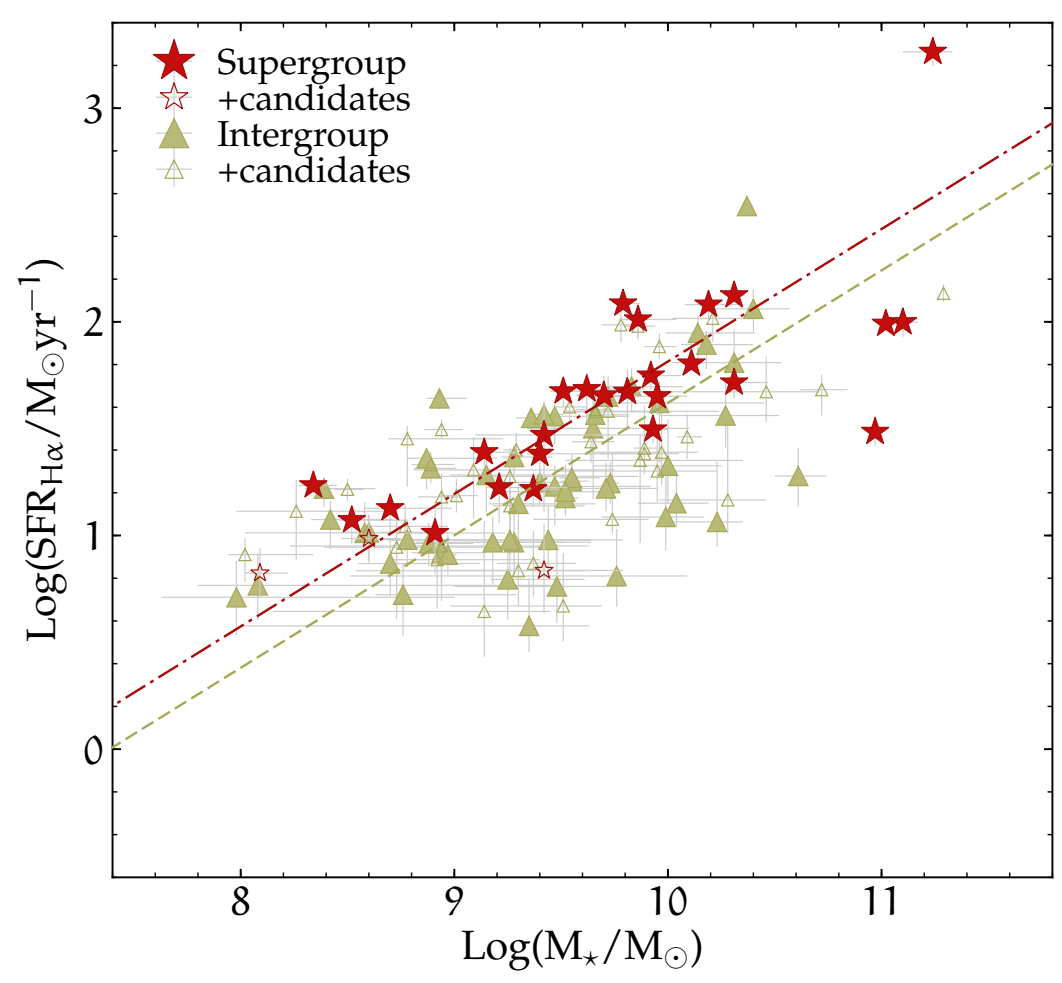

Figure 6. The same as Fig. 5, but here we compare HAEs in between the supergroup (top quartile of overdensities) shown by red star symbols and the intergroup regions. Red dash-dot and dashed lines are the median-fitted lines for supergroup and intergroup HAEs assuming the same slope as the entire HAEs, respectively.

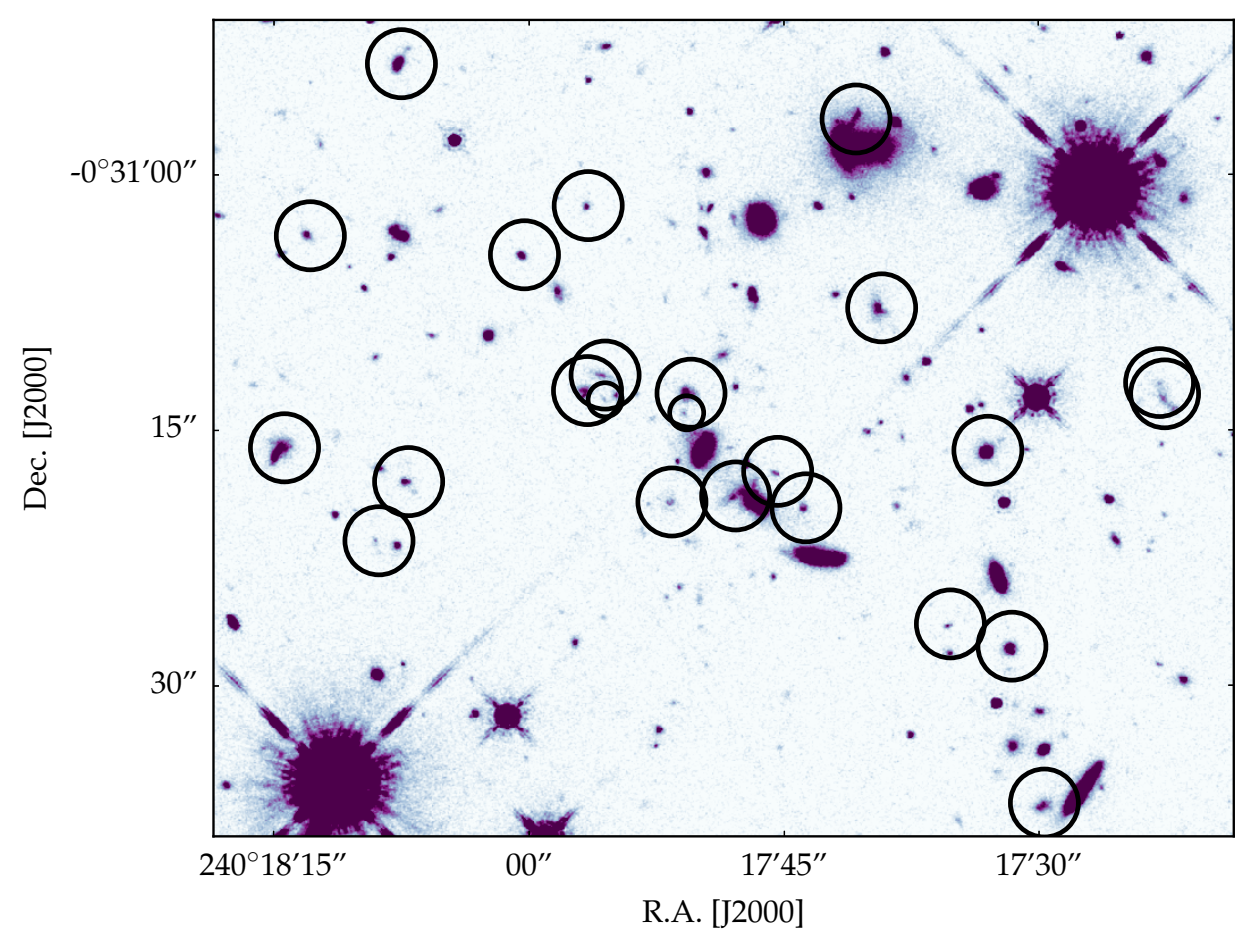

Figure 7. F160W image form WFC3/HST $\left(1.0 \times 0.8 \operatorname{arcmin}^{2}\right)$ around the peak density in the USS 1558 protocluster. Large and small circles indicate HAEs and HAE candidates selected by this work, respectively. 


\subsection{Mass-size distribution}

Size estimation of HAEs in the protocluster and comparison to stellar sizes across environments allow an investigation of the environmental effects on size growth histories at high redshifts. For example, overdense environments may involve the higher chance of galaxy mergers (Okamoto \& Habe 2000; Gottlöber et al. 2001; Hine et al. 2016). Such merger events will change the size growth rate as a function of total stellar mass. Specifically, it is expected that major mergers and minor mergers increase the effective radii $R_{\boldsymbol{e}}$ of stellar components scaled as $\delta \log \left(R_{e}\right) / \delta \log \left(\mathrm{M}_{\star}\right) \sim 1$ and 2 , respectively (Bezanson et al. 2009; Naab et al. 2009; van Dokkum et al. 2010). Mergers would also drive the gas towards the centre and may induce more centrally concentrated star formation (e.g. Mihos \& Hernquist 1996; Teyssier et al. 2010; Bournaud et al. 2015). So far, many studies have investigated the environmental dependence of mass and size evolution in high- $z$ clusters (Papovich et al. 2012; Lani et al. 2013; Gobat et al. 2013; Newman et al. 2014; Belli et al. 2014; Tran et al. 2017; Kubo et al. 2017), however, the conclusion is still controversial, especially in rich systems of star-forming galaxies like USS 1558. The existing F160W image in USS 1558 taken by HST (Fig. 7) allows us to derive the sizes of rest-frame optical light for the HAEs, based on the model profile fitting technique by GALFIT (Peng et al. 2010a).

We estimate effective radii $R_{\boldsymbol{e}}$ of HAEs from the F160W image by using the GALFIT version 3.0 code (Peng et al. 2010a). The F160W filter does not include strong $\mathrm{H} \beta$ and [OіII] $\lambda \lambda 4960,5008$ emission lines, and thus, the derived $R_{\boldsymbol{e}}$ should be tracing only stellar components of the target HAEs. Since our HST observation mainly focused on the overdense regions (Hayashi et al. 2016), only 47 (1) and 12 (7) bright HAEs (or HAE candidates) with F160W $<25$ mag (corresponds to $\gtrsim 10 \sigma$ detections) are available. Initial parameters for the model fitting (i.e. central position, total magnitude, half light radius, axis ratio, and position angle) incorporate the results from SExtractor (Bertin \& Arnouts 1996). We initially set a Sersic index of 1.5 , and allow it to be $0.2-8$ in the fitting. We employed the PSF model from the 3D-HST (Skelton et al. 2014) that has better signal-to-noise ratio than that of the PSF model made by the composite image of bright stars in our survey field. In addition, we correct the wavelength dependence of size estimation caused by radial colour gradients of galaxies that largely depend on stellar mass (Wuyts et al. 2012; van der Wel et al. 2014) according as the empirical relation provided by van der Wel et al. (2014), which converts the obtained effective radii $\left(R_{\boldsymbol{e}}\right)$ to that at the rest frame wavelength of $5000 \AA$. This empirical conversion is applicable only for star-forming galaxies, which can be eligible for most HAEs. One should note that the correction factors are at most 4 per cent for our sample, which are negligibly small. Our size measurement includes photometric errors coupled with the fitting errors from GALFIT, but does not consider further possible uncertainties as noted by van der Wel et al. (2012). Therefore, our measured $R_{\boldsymbol{e}}$ may have additional errors of at most 0.3 dex depending on these actual surface brightness and radial profiles (van der Wel et al. 2012).

The obtained results are shown in Fig. 8 and summarised in Table A. Unfortunately, the sample size in the intergroup regions is obviously insufficient, and thus, we can only make a poor comparison between two populations of different densities. We also estimate offsets from the masssize relation (Shen et al. 2003) of star-forming galaxies at $z=2-2.5$ reported by van der Wel et al. (2014), which is defined by $\Delta R e_{V 14}$. We obtained those typical values of $\Delta R e_{V 14}=0.15$ and 0.08 dex in group HAEs and intergroup HAEs. We cannot identify the apparent deviation of size distributions in our samples, since those still fall within $1 \sigma$ scatter $(\sim 0.19$ dex $)$ of van der Wel et al. (2014), respectively. We also confirm that the stellar size distribution of our HAEs is consistent with that of HAEs in general field at $z=2.2$ (Stott et al. 2013). According to the KS test, we cannot rule out that groups of HAEs belong to the same parent sample as those in the intergroup regions along the mass-size relation ( $p=0.379$, see $\S 3.5)$. In fact, rather, it is hard to judge how our HAEs differ in mass-size distribution within our sample and galaxies in the previous studies due to restricted sample size. Furthermore, our small sample cannot constrain any relation between stellar mass and effective radius with the high $p$-value of 0.15 by the Spearman's rank correlation coefficient. Considering these factors, our data should not be enough to discuss size evolution and we decide not to delve into the size distribution in this paper.

On the other hand, we identify two and one compact HAEs in the higher and lower densities in the protocluster, respectively, that follow the mass-size relation of early type galaxies at the similar redshift slice (van der Wel et al. 2014). It would be intriguing to carry out follow-up spatiallyresolved analyses of those star-forming activities based on integral field spectroscopy of $\mathrm{H} \alpha$ line or dust continuum to understand the formation mechanisms of passive red sequence objects in local galaxy clusters.

\section{4 $\mathrm{H} \alpha$ luminosity and stellar mass functions}

It is also essential to compare number distributions of such physical properties across overdensities, since we can expect that those distribution functions would depend on surrounding environments inferred from the substantial excess of very massive quiescent galaxies in the cluster centre in the local Universe. Indeed, previous studies have shown the enhancement of massive star-forming galaxies in protoclusters as compared to the general fields (Steidel et al. 2005; Koyama et al. 2013b). Our extended HAE sample allows us to quantify and advance such past achievements by determining number distributions as a function of $\mathrm{H} \alpha$ luminosity and stellar mass based on the Schechter (1976) function.

The key issue in a derivation of distribution functions is the completeness correction (e.g. Sobral et al. 2009, 2013, 2014). We correct the numbers of the HAE sample based on their narrowband magnitudes as described in Appendix B, which includes the correction of both detection and selection completeness derived by the Monte Carlo simulation. Our procedure is similar to the technique by Sobral et al. (2009, 2013) that have conducted completeness correction in $\mathrm{H} \alpha$ selected galaxies by narrowband imaging surveys.

However, we should still miss a bunch of HAEs due to the incompleteness of the following colour-colour selections that were conducted to remove other line contaminants (§2.3). This additional colour selection produced 51 unclassified narrowband emitters defined as HAE candidates (Table 2), which are a lack of photometry of at least one band 

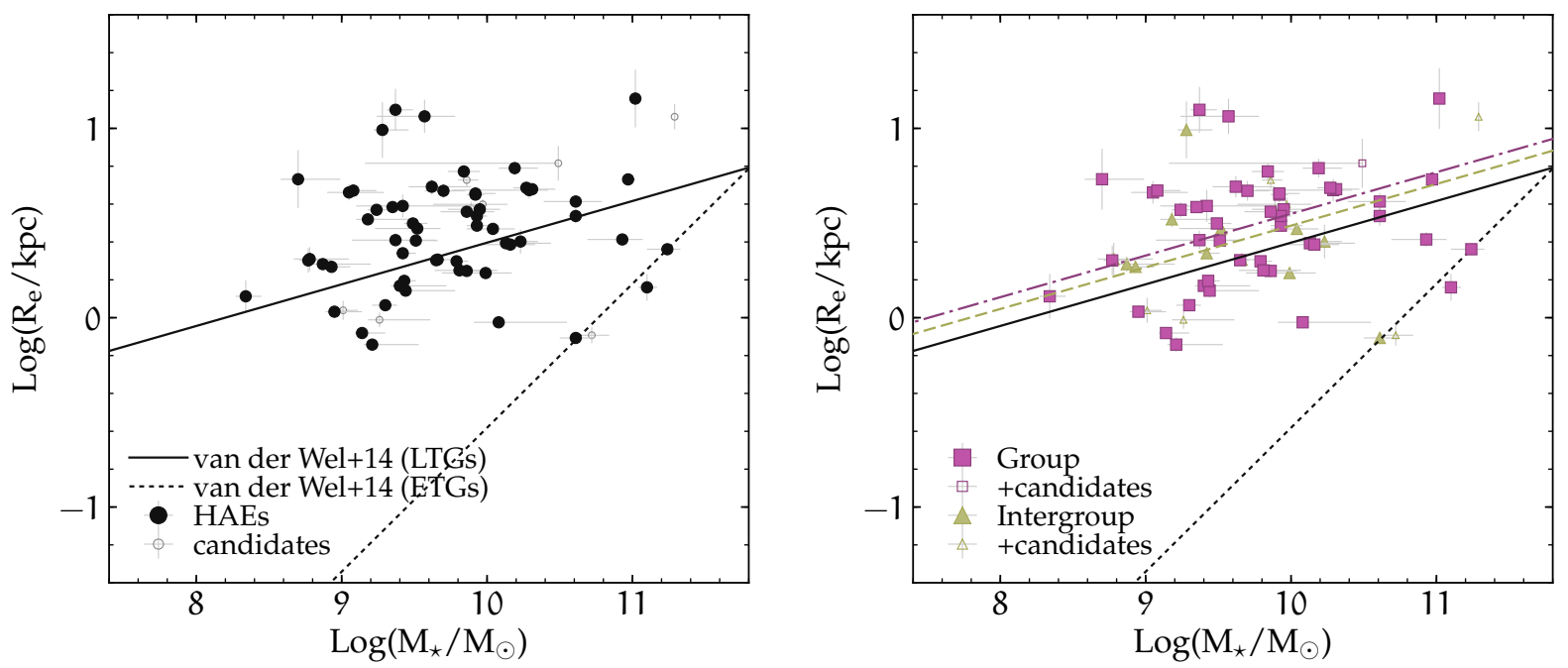

Figure 8. Stellar mass versus effective radius. Two panels show the same results, but symbols are changed by different environments in the right figure. The symbols are the same as shown in Fig. 5. Dashed and dash-dot lines indicate mass-size relation of star-forming galaxies and quiescent galaxies reported by van der Wel et al. (2014). In the right panel, purple dash-dot and yellow dashed lines show the median-fitted lines under the same slope of the black solid line. PSF objects are estimated to be $\log \left(\mathrm{R}_{\mathrm{e}} / k_{p} c\right) \sim-0.7$ dex.

among $B, r^{\prime}, J, K s$ filter bands. Since 86 per cents of those are low-mass objects with stellar masses lower than $10^{10} \mathrm{M}_{\odot}$, the colour-colour selection processes particularly miss the objects at the faint end. To minimise this impact, we compromise by adding a correction of 15 per cent contaminations for the number of HAE candidates (§2.3). We then use the combined sample of HAEs and HAE candidates for the fitting of the Schechter function. This control sample should provide us with a relatively-complete HAE sample that is less affected by the colour-colour selection criteria, and enable fairer results for our analyses. One should note that our correction process cannot recover HAEs with $\mathrm{EW}_{\mathrm{H} \alpha}$ lower than $45 \AA$ in the rest frame.

Figure 9 shows the distribution functions for $\mathrm{H} \alpha$ luminosity and stellar mass of HAEs. As described above, we employed the combined sample of HAEs and HAE candidates, and we also show only the HAE in each panel. We have performed the non-linear least chi-square fitting by the Schechter function to the entire HAEs, and HAEs in group and intergroup environments, with the MPFIT package (Markwardt 2009) ${ }^{4}$. The MPFIT fitting code semiautomatically provides us with the best fitting parameters and errors. We remove the faintest and two brightest bins in the fitting process for $\mathrm{H} \alpha$ luminosity function since the former has a significant completeness issue and the the brightends may have a large contribution by AGN (Matthee et al. 2017) (and indeed the brightest bin includes only RG). Also, we reject the stellar mass bins of $<10^{9.4} \mathrm{M}_{\odot}$ for the fitting of stellar mass function since the SFR limit causes a critical incompleteness problem for the lower-mass HAEs than this threshold along the star-forming main sequence (§3.2). The sample bins used in the curve fitting are highlighted by the thick frames in each panel.

The results of the Schechter function fitting are available in Table 3. The Schechter function is given by the fol-

${ }^{4}$ http://purl.com/net/mpfit lowing equations,

$$
\begin{aligned}
\phi(L) d L & =\phi^{*}\left(\frac{L}{L^{*}}\right)^{\alpha} \exp \left(-\frac{L}{L^{*}}\right) \frac{d L}{L^{*}} \\
\phi(L) d L & =\phi^{*}\left(\frac{L}{L^{*}}\right)^{\alpha+1} \exp \left(-\frac{L}{L^{*}}\right) \ln 10 d(\log L),
\end{aligned}
$$

where $L$ is $\mathrm{H} \alpha$ luminosity or stellar mass in this work. $L^{*}$ is the characteristic luminosity or stellar mass in which the power-law slope cuts off. We count the number of galaxies as follows,

$\phi(\log L)=\Sigma_{i}\left(V_{\max } \cdot C(N B) \cdot \Delta(\log L)\right)^{-1}$,

where $V_{\max }\left(\mathrm{co}-\mathrm{Mpc}^{3}\right)$ is the volume size derived from the filter FWHM of the narrowband filter $(45 \mathrm{co}-\mathrm{Mpc})$ and survey area $\left(58 \mathrm{co}-\mathrm{Mpc}^{2}\right)$. Those of the group and the intergroup regions can be estimated to be 609 and $3802 \mathrm{co-Mpc}{ }^{3}$, respectively. Actual volume size should be smaller than those values since HAEs are concentrated around the protocluster centre according to Shimakawa et al. (2014). We ignore this systematic error since we solely compare the shape of distribution functions between higher and lower densities within the same protocluster region. Due to the small sample size, we have to fix the power-law slope $\alpha=-1.6$ for the $\mathrm{H} \alpha$ luminosity function and $\alpha=-1.5$ for the stellar mass function, respectively, to minimise the fitting errors. These $\alpha$ values are employed from Sobral et al. (2013) and Davidzon et al. (2017), respectively. In either case, we also tried differing $\alpha$ by \pm 0.1 , and the result did not improve. We should keep in mind that our restricted sample sizes may be insufficient to determine the robust function parameters and even errors. For example, even if one has sufficient statistics on large datasets, there are variations of obtained $M_{\star}^{*}$ and $\Phi^{*}$ due to sample variance and selection effects (Ilbert et al. 2013; Muzzin et al. 2013; Sobral et al. 2014; Davidzon et al. 2017; Hayashi et al. 2017a). We believe that systematic comparisons between different environments given only our data would work well. To compare our samples with results from the literature would require far more attention as they 

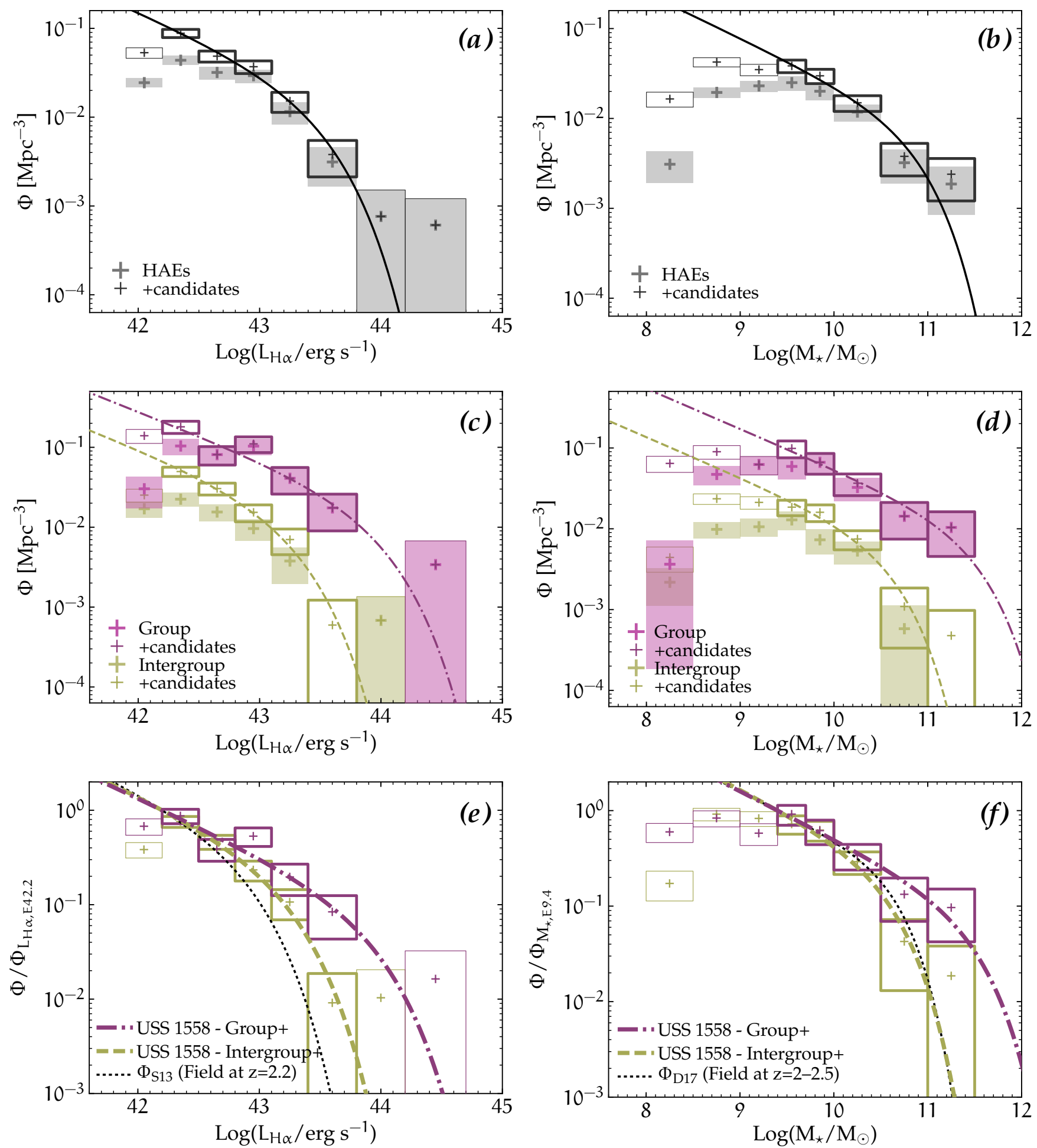

Figure 9. H $\alpha$ luminosity (left) and stellar mass (right) distributions of our HAE samples. Filled boxes indicate the HAE sample and open ones include HAE candidates with 15 per cent contamination correction. (a,b) show the entire protocluster region, and (c,d,e,f) represent the dense groups (purples) and the intergroup regions (yellows). In the panel (e,f), the H $\alpha$ luminosity and stellar mass functions are normalised by the values at $L_{\mathrm{H} \alpha}=10^{42.2} \mathrm{erg} \mathrm{s}^{-1}$ and $\mathrm{M}_{\star}=10^{9.4} \mathrm{M}_{\odot}$, respectively. Black solid, purple dash-dot, and yellow dashed curves are best-fitted Schechter functions for the protocluster, group, and intergroup samples, respectively. The fitting results and the scaling factors are listed in Table 3. Black dotted curves in (e) and (f) indicate the distribution functions of HAEs at $z=2.2$ in the random fields by Sobral et al. (2013) and star-forming galaxies at $z=2-2.5$ in the random fields by Davidzon et al. (2017), respectively. 
Table 3. Results of Schechter function fitting for $\mathrm{H} \alpha$ luminosity and stellar mass bins. The first column specifies the sample. The second and fifth columns show the characteristic $\mathrm{H} \alpha$ luminosity and stellar mass, respectively. The third and sixth columns represent normalisations for $\mathrm{H} \alpha$ luminosity and stellar mass, respectively. Our line fitting assumes the fixed slope $\alpha=-1.6$ and -1.5 for $\mathrm{H} \alpha$ luminosity and stellar mass, respectively. The fourth and seventh columns show the scaling factor used when we normalise and then compare those with each other (bottom panels in Fig. 9). The stellar mass function for the group HAE sample can be also fitted by the following power-law function, $\log \left(\Phi_{\mathrm{M}_{\star}} / \mathrm{Mpc}^{-3}\right)=3.696 \pm 0.063-0.5 \times \log \left(\mathrm{M}_{\star} / \mathrm{M}_{\odot}\right)$.

\begin{tabular}{lcccccc}
\hline Sample & $\log \left(L_{\mathrm{H} \alpha}^{*} / \mathrm{erg} \mathrm{s}^{-1}\right)$ & $\log \left(\Phi_{\mathrm{H} \alpha}^{*} / \mathrm{Mpc}^{-3}\right)$ & $\Phi_{\mathrm{H} \alpha=\mathrm{E} 42.2} / \Phi_{\mathrm{S} 13}$ & $\log \left(\mathrm{M}_{\star}^{*} / \mathrm{M}_{\odot}\right)$ & $\log \left(\Phi_{\mathrm{M}_{\star}}^{*} / \mathrm{Mpc}^{-3}\right)$ & $\Phi_{\mathrm{M}_{\star}=\mathrm{E} 9.4} / \Phi_{\mathrm{D} 15}$ \\
\hline All & $43.466 \pm 0.120$ & $-2.056 \pm 0.102$ & 14.1 & $10.955 \pm 0.178$ & $-2.464 \pm 0.112$ & 14.0 \\
Group & $43.937 \pm 0.441$ & $-2.079 \pm 0.309$ & 26.7 & $11.505 \pm 0.588$ & $-2.380 \pm 0.341$ & 31.6 \\
Intergroup & $43.207 \pm 0.110$ & $-2.105 \pm 0.109$ & 8.5 & $10.603 \pm 0.179$ & $-2.529 \pm 0.145$ & 7.5 \\
\hline
\end{tabular}

have used different sample selections based on the different datasets.

To make fairer comparisons in various environments, we also show both $\mathrm{H} \alpha$ luminosity and stellar mass functions normalised by those faint-end values of the employed sample bins, $\log \left(L_{\mathrm{H} \alpha} / \mathrm{erg} \mathrm{s}^{-1}\right)=42.2$ and $\log \left(\mathrm{M}_{\star} / \mathrm{M}_{\odot}\right)=9.4$, respectively. These scaled distributions appear in the bottom of Fig. 9, and scaling values can be found in Table 3 that would be useful to quantify the overdensities of our targets more generally.

The derived distribution functions of the two environments show significant differences in the sense that the dense groups tend to host a larger number of more massive and more active HAEs than the intergroup regions within the same protocluster field. The results suggest that environmental effects on the scale of $<1$ ph-Mpc change the physical process or time-scale of galaxy formation in this young protocluster. Both $L_{\mathrm{H} \alpha}^{*}$ and $\mathrm{M}_{\star}^{*}$ in HAEs in the dense group cores are significantly higher than those in the intergroup regions by 0.7 and 0.9 dex, respectively. When we compare the $\mathrm{H} \alpha$ luminosity functions with that for the random field (Sobral et al. 2013), the dense groups exhibit a much larger number of HAEs at higher luminosities, and there may be a slight excess in the intergroup environments. According to the comparison of the scaled fitting curves, for example, the dense groups host by eight times larger number of HAEs at $\mathrm{M}_{\star}=10^{11} \mathrm{M}_{\odot}$ than the intergroup regions.

On the other hand, interestingly, the intergroup regions likely show the very similar shape of stellar mass function as that of star-forming galaxies in the general field at similar redshifts (Davidzon et al. 2017). Not only derived mass function, but also those stellar mass bins are well consistent with Davidzon et al. (2017), suggesting non-environmental dependence of stellar mass distribution outside of the densest regions despite that the intergroup regions are also associated with the same dense protocluster, and have eight times higher densities on average than the general fields. However, we should note that stellar mass function of Davidzon et al. (2017) has a lower cutoff than those of the past studies (Ilbert et al. 2013; Muzzin et al. 2013; Sobral et al. 2014), and thus our comparison result changes depending on the literature and the power slope $(\alpha)$ used for the line fitting. To resolve this issue, more comprehensive analyses in both protoclusters and general fields should be required.

On top of that, the faint end profiles of distribution functions should be another intriguing point. Environmental dependence of stellar mass function in the low-mass end at low redshifts has been widely studied (Trentham 1998;
Conselice 2002; Yagi et al. 2002; De Propris et al. 2003; Penny \& Conselice 2008), especially toward a better understanding of the environmental quenching (Peng et al. 2010b; Bolzonella et al. 2010; Mortlock et al. 2015). At higher redshift, $z \sim 1$, Sobral et al. (2011) and Tomczak et al. (2017) find the flatter slope at the faint end in higher local overdensities. However, the faint end slope of star-forming galaxies in high- $z$ protoclusters at $z>2$ has been poorly understood. Cooke et al. (2014) have reported a steeper $\alpha$ in the stellar mass distribution in protocluster regions based on the Millennium Simulation (Springel et al. 2005). On the other hand, as also mentioned by Cooke et al. (2014), high densities could involve environmental quenching in the large host haloes at even such high redshifts. Regrettably, the current datasets cannot constrain the power-law slope $\alpha$ for both $\mathrm{H} \alpha$ and stellar mass functions due to the small sample size. However, judging from the comparisons of the normalised distribution functions, we cannot see the clear distinctions from each other at the faint end of these functions. The much larger datasets must be needed to improve the statistics and quantify the faint end slopes more precisely.

\subsection{Environmental dependence within the protocluster}

We here summarise the comparison tests of physical properties between HAEs in lower and higher densities selected based on the 5 th mean projected distance $\left(\bar{b}_{5 \text { th }} \gtrless 220\right.$ ph$\mathrm{kpc})$ across the survey field. We test if there are any statistical differences between two groups by using the KS test in nine properties: (a) stellar mass, (b) SFR, (c) sSFR, (d) deviation from the main sequence $(\Delta \mathrm{MS}),(\mathrm{e})$ dust reddening derived from the SED-fitting, (f) offset from the stellar mass $-\mathrm{E}(\mathrm{B}-\mathrm{V})$ relation $(\Delta \mathrm{E}(\mathrm{B}-\mathrm{V})$, see Fig. 4$)$, (g) effective radius, (h) deviation from the mass-size relationship by van der Wel et al. (2014) $\left(\Delta \mathrm{Re}_{\mathrm{V} 14}\right)$, and (i) J - Ks colour striding over Balmer $/ 4000 \AA$ break.

Obtained $p$-values from the KS test and median values in each physical parameter are available in Table 4 and Fig. 10. Table 4 also shows the results of the KS test between HAEs in the supergroup and the intergroup regions. The comparisons of those cumulative distribution functions can be seen in Fig. 11 for the former six parameters that show different $p$-values as compared to those between the group and the intergroup regions. These results address environmental effects for galaxy formation in the extreme. The comparison results show statistically different between group and intergroup HAEs in SFR, E(B-V), and effective ra- 

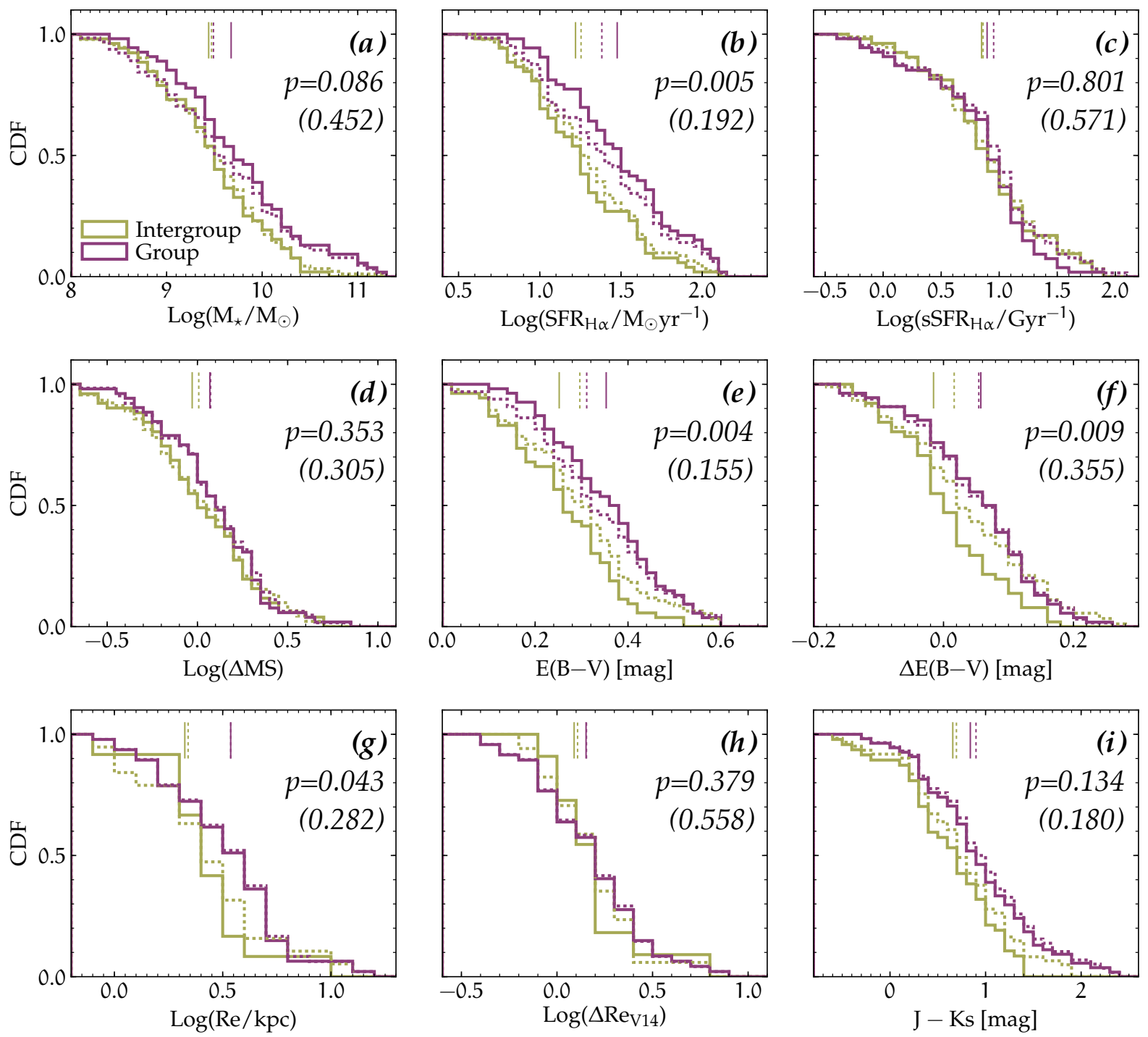

Figure 10. Cumulative distribution functions (CDFs) for galaxies properties, (a) stellar mass, (b) SFR, (c) sSFR, (d) deviation from the main sequence, $\triangle \mathrm{MS}$, (e) SED-inferred stellar extinction $\mathrm{E}(\mathrm{B}-\mathrm{V})$, (f) offsets from the mass-E(B-V) sequence of the entire HAEs, (g) effective radius, $\boldsymbol{R}_{\boldsymbol{e}},(\mathrm{h})$ offset from the mass-size relation of star-forming galaxies by van der Wel et al. (2014) $\left(\Delta \mathrm{Re}_{\mathrm{V} 14}\right)$, and (i) $\mathrm{J}-\mathrm{Ks}$ colour striding Balmer/4000A break. Purple and yellow lines indicate CDFs of HAE in group region and intergroup region, which are divided by the median values of the mean projected separation of $220 \mathrm{ph}-\mathrm{kpc}$. Solid lines show the HAE sample and dotted lines include HAE candidates. The vertical lines in each panel show the median values of respective samples (Table 4).

Table 4. The results of the KS test for nine physical parameters listed in the first column. The second-fourth columns show median values of each parameter of HAEs in the group (50th \%tile higher densities), the intergroup (50th \%tile lower densities), and the supergroup regions (top quartile higher densities), respectively. The fifth and sixth columns indicate $p$-values of the KS test for each physical parameter. Values enclosed in parentheses are based on the sample including HAE candidates.

\begin{tabular}{lccccc}
\hline Parameter & Grõup & Interg̃roup & Supergroup & $p_{\text {(Group-Intergroup) }}$ & $p_{\text {(Supergroup-Intergroup) }}$ \\
\hline $\log \left(\mathrm{M}_{\star} / \mathrm{M}_{\odot}\right)$ & $9.68(9.49)$ & $9.44(9.47)$ & $9.80(9.70)$ & $0.086(0.452)$ & $0.081(0.287)$ \\
$\log \left(\mathrm{SFR} / \mathrm{M}_{\star} \mathrm{yr}^{-1}\right)$ & $1.48(1.38)$ & $1.22(1.25)$ & $1.66(1.65)$ & $0.005(0.192)$ & $0.0003(0.002)$ \\
$\log \left(\mathrm{sSFR} / \mathrm{Gyr}^{-1}\right)$ & $0.90(0.95)$ & $0.85(0.86)$ & $0.97(0.98)$ & $0.801(0.571)$ & $0.319(0.388)$ \\
$\log (\Delta \mathrm{MS})$ & $0.07(0.07)$ & $-0.03(0.01)$ & $0.16(0.18)$ & $0.353(0.305)$ & $0.030(0.044)$ \\
$\mathrm{E}(\mathrm{B}-\mathrm{V})$ & $0.35(0.31)$ & $0.25(0.30)$ & $0.38(0.38)$ & $0.004(0.155)$ & $0.0006(0.019)$ \\
$\Delta \mathrm{E}(\mathrm{B}-\mathrm{V})$ & $0.06(0.05)$ & $-0.02(0.02)$ & $0.07(0.06)$ & $0.009(0.355)$ & $0.004(0.306)$ \\
$\log (\mathrm{Re} / \mathrm{kpc})$ & $0.54(0.54)$ & $0.33(0.34)$ & $0.55(0.55)$ & $0.043(0.282)$ & $0.049(0.334)$ \\
$\log \left(\Delta \mathrm{Re}_{\mathrm{V} 14}\right)$ & $0.15(0.15)$ & $0.09(0.11)$ & $0.14(0.14)$ & $0.379(0.558)$ & $0.468(0.769)$ \\
$\mathrm{J}-\mathrm{Ks}$ & $0.84(0.90)$ & $0.66(0.82)$ & $0.92(0.99)$ & $0.134(0.180)$ & $0.100(0.102)$ \\
\hline
\end{tabular}



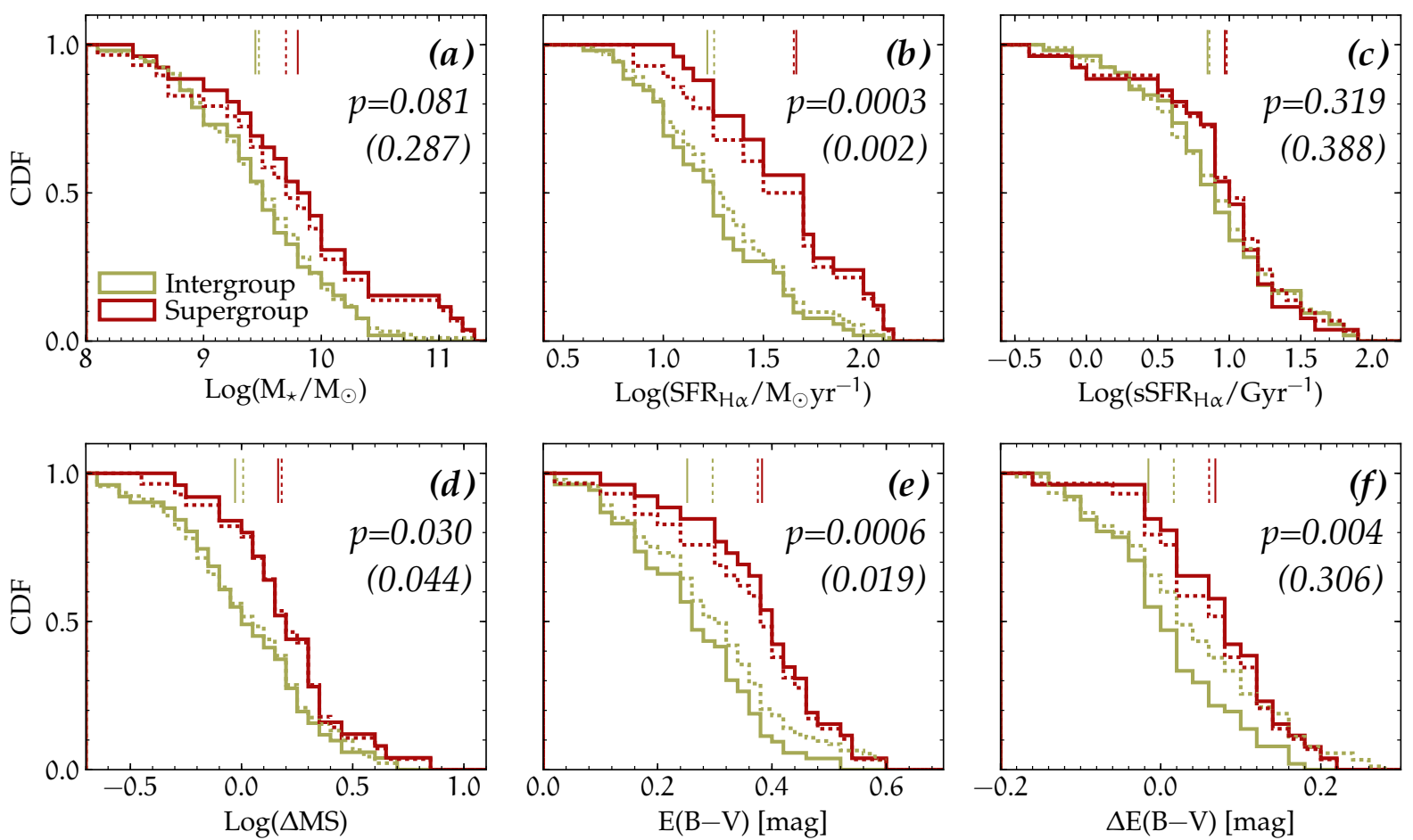

Figure 11. Cumulative distribution functions (CDFs) for galaxies properties, (a) stellar mass, (b) SFR, (c) sSFR, (d) $\triangle \mathrm{MS}$, (e) E(B-V), and (f) $\triangle \mathrm{E}(\mathrm{B}-\mathrm{V})$ of HAEs in the supergroup (reds) and the intergroup (yellow) regions. The solid line shows the HAE sample and dotted line include HAE candidates. The vertical lines in each panel show the median values of respective samples.

dius, with $p$-values lower than 5 per cent. These suggest that HAEs in the dense groups tend to have higher SFRs, larger amounts of dust extinctions, and larger stellar sizes, respectively. As mentioned in $\S 3.2$ and $\S 3.3$.

However, such trends seen in SFR and effective radius can no longer be confirmed for a given stellar mass, according to the comparisons for $\Delta \mathrm{MS}$ and $\Delta \mathrm{Re}_{\mathrm{V} 14}$, respectively. Indeed, such SFR and size differences between group and intergroup regions are supposed to be caused by the significant excess of massive HAEs in the dense cores, as provided by these stellar mass function (Fig. 9). On the other hand, the statistical difference seen in the dust reddening of HAEs remains consistent with $p=0.009$ if we compare those for a given stellar mass $(\Delta \mathrm{E}(\mathrm{B}-\mathrm{V})$ in Table 4$)$ along the fitted line of the mass-extinction relation (Fig. 4). Such higher dust obscuration of galaxies in local overdensities for a given stellar mass range is consistent with the results of past studies (Koyama et al. 2013b; Hatch et al. 2017) that have investigated the environmental dependence of dust reddening of galaxies on a more global scale. However, our measurement of the dust reddening is based on the SED-fitting under the assumption of fixed stellar metallicity and limited star formation history. Therefore, such a systematic difference could be also interpreted by difference in metal abundance, age, and tau between the two samples, which is actually suggested by the previous studies (e.g. Ferré-Mateu et al. 2014; Genel 2016). It should be noted that at least we could not find clear differences in the SED-inferred age and tau between HAEs in higher and lower densities. To resolve this problem, we need more direct estimation, e.g. Balmer decrement technique based on $\mathrm{H} \alpha$ and $\mathrm{H} \beta$ line spectra (Shivaei et al. 2015).
Furthermore, HAEs in extremely high (top quartile) overdensities categorised as the supergroup, show more discriminable properties as compared to those in the intergroup regions. The KS test shows even lower $p$-values in the comparisons of most physical properties, and we for the first time identify that the supergroup HAEs tend to have higher SFRs across the star-forming main sequence than the intergroup HAEs. As inferred from the margin of those median $\Delta \mathrm{MS}$ values of $0.19 \mathrm{dex}$, stellar mass assembly in the supergroups are by 1.5 times accelerated as a function of stellar mass, which may largely contribute to the excess of massive HAEs in local overdensities seen in the stellar mass functions. Such a prominent deviation of the main sequence in higher densities has not been found in the past studies (Koyama et al. 2013a,b). They have investigated the environmental dependence of star-forming activities on a more global scale based on shallower data than this paper. Such different observing scale and survey depth may be one of the primary reasons of the discrepancy. Indeed, our previous shallower data in the same USS 1558 field show no significant $\Delta$ MS enhancement in the densest group region (Hayashi et al. 2012), suggesting sufficiently deep $\mathrm{H} \alpha$ imaging is important to identify enhanced star formation in the extremely dense groups. Moreover, differences in the forming phase of the clusters can be another key factor, since for example, our target USS 1558 at $z=2.5$ is apparently younger protocluster system than PKS 1138-262 at $z=2.2$ studied by Koyama et al. (2013a,b) in the sense of less established red sequence, more clumpy substructure, and higher redshift in USS 1558 relative to PKS 1138. We discuss more detailed explanations on the physical origins of the SFR enhancements in the discussion section $(\S 4)$. 
Lastly, we cannot identify the clear difference in the other physical properties shown in Fig. 10. First, this work has found the significant enhancement of massive HAEs in the dense cores. However, mass distributions are consistent within errors between the group and the intergroup regions at the stellar mass lower than $10^{10.5} \mathrm{M}_{\odot}$, and thus, the difference of mass distributions is no longer significant in the entire mass range. Also, we cannot rule out the fact that size distributions do not depend on local overdensities for a given stellar mass because of high $p$-values $(>0.05)$ in the comparisons of $\Delta \operatorname{Re}_{\mathrm{V} 14}$ (see also §3.3). This means that early environmental effects in the protocluster may solely affect the stellar mass assembly histories (and probably dust reddening) of galaxies, but have little impact on those size growth histories. Besides, our results show no clear difference in Balmer $/ 4000 \AA$ colour $(\mathrm{J}-\mathrm{Ks})$. In USS 1558, a tight red sequence is not established yet unlike the more advanced protocluster systems at $z \sim 2$ (Kodama et al. 2007), which may be the reason why we cannot see the significant excess of redder HAEs in the dense cores.

\section{DISCUSSION}

Using a total of $107 \mathrm{HAE}$ sample and 51 additional HAE candidates, this work has analysed the environmental dependence of physical properties of $\mathrm{H} \alpha$-emitting galaxies within the protocluster on the substructure $(<1 \mathrm{ph}-\mathrm{Mpc})$ scale. Despite such a small scale, we find a significant enhancement of more massive and active HAEs in the densest parts of the protocluster. We discuss potential physical mechanisms that drive these observational tendencies and try to explain galaxy formation in young star-forming protoclusters like our target USS 1558, together with previous observational and theoretical work.

Firstly, the comparison of cumulative distribution functions reveals a statistical difference in SFRs of HAEs in between lower and higher densities, which would be independent of uncertainties of estimation of dust reddening since this tendency still can be confirmed without dust correction. Figure 12 represents the characteristic $\mathrm{H} \alpha$ luminosities $\left(L_{\mathrm{H} \alpha}^{*}\right)$ as a function of redshift for our samples and field galaxies (Sobral et al. 2013; Hayashi et al. 2017a). These are derived based on the Schechter function assuming a fixed $\alpha=-1.6$, which allows us to make a relatively fair comparison of the cutoff $\mathrm{H} \alpha$ luminosities of the power-law form among these samples. Also, one should note that all of the reference samples are based on narrowband imaging surveys.

As seen in the figure, HAEs in the intergroup region of USS 1558 follow the extrapolation of the redshift evolution of the characteristic $\mathrm{H} \alpha$ luminosity reported by Sobral et al. (2013), while HAEs in dense group environments deviate from the sequence. This suggests that the densest environment in the young protocluster have a larger number of active star-forming galaxies than the intergroup regions in the same protocluster field. Such higher cutoff $\mathrm{H} \alpha$ luminosity in overdensities were not explored in Hatch et al. (2011) for a protocluster PKS 1138-262 at $z=2.2$. However, the additional $\mathrm{H} \alpha$ imaging for the same field has found a number excess of massive HAEs with higher SFRs along the star-forming main sequence (Koyama et al. 2013a,b), suggesting that this trend might be a common feature during

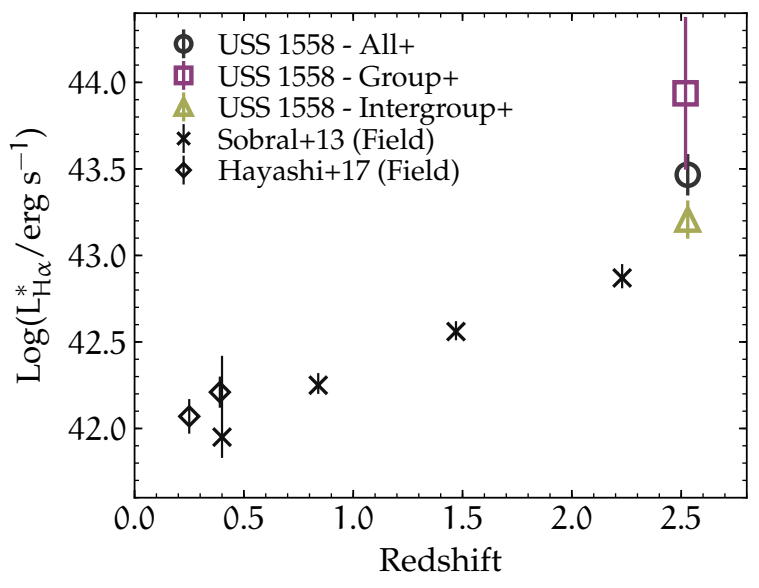

Figure 12. The characteristic $\mathrm{H} \alpha$ luminosity $\left(L_{\mathrm{H} \alpha}^{*}\right)$ as a function of redshift. Purple square, black circle, and yellow triangle are based on our sample in the entire protocluster, group, and intergroup regions in USS 1558 at $z=2.53$, respectively. The purple square symbol is a bit shifted leftward to improve visualisation. Crosses and diamonds are $L_{\mathrm{H} \alpha}^{*}$ derived from the similar $\mathrm{H} \alpha$ survey for the general fields at lower redshifts by Sobral et al. (2013) and Hayashi et al. (2017a), respectively. Errorbars show $1 \sigma$ fitting errors.

rapid building of bright red sequence objects in the protocluster. We should note, however, environmental dependence of galaxy properties will depend on their evolutionary phase of each protocluster system.

The bright-end HAEs in $\mathrm{H} \alpha$ luminosity function may host active galactic nuclei (AGN). Sobral et al. (2016) find a tight correlation between $\mathrm{H} \alpha$ brightness and AGN fraction, which for example shows 50 per cent AGN fraction in HAEs with $L_{\mathrm{H} \alpha}=5 \times L_{\mathrm{H} \alpha}^{*}$ (see also Matthee et al. 2017). Thus, taking account of the potential increase of AGN fraction in high- $z$ protoclusters (Lehmer et al. 2009, 2013), we could explain the higher cutoff value of $\mathrm{H} \alpha$ lunminosity function in the dense groups by a substantial excess of AGN. However, we see the number excess of HAEs near $L_{\mathrm{H} \alpha}^{*}$ and such a significantly luminous HAE $\left(L_{\mathrm{H} \alpha} \sim 10^{44} \mathrm{erg} \mathrm{s}^{-1}\right)$ is only the radio galaxy in our sample. Thus, it may seem to be difficult to examine the higher characteristic $\mathrm{H} \alpha$ luminosity only by the substantial excess of AGNs in the group environments. Future deep follow-up X-ray and near-infrared spectroscopy will help us to test the connection between AGN activities and environmental dependence of the luminosity function.

We also identify a prominent enhancement of massive HAEs $\left(M_{\star}>10^{10.5} M_{\odot}\right)$ in the dense groups by comparing those stellar mass distribution with that of the intergroup HAEs. This is broadly consistent with Steidel et al. (2005); Koyama et al. (2013a,b) who reported similar trends by comparing protocluster and field galaxies, while we find the excess of massive HAEs in substructures within the protocluster. It is crucially important to understand characteristics of those massive $\mathrm{H} \alpha$-emitting galaxies to infer the physical origins of the strong environmental dependencies seen in the local Universe, since they would be the progenitors of early-type galaxies seen at the bright end of the red sequence seen in today's galaxy clusters (Gladders \& Yee 2000; Thomas et al. 2005; Koester et al. 2007; Stott et al. 2009; Cappellari et al. 2011). However, the current relevant 
datasets seem to argue that most of massive HAEs except the RG are likely star-forming galaxies rather than AGNs, for example, because those rest optical line spectra available in five out of six objects do not show strong [NII] emission line features (Shimakawa et al. 2015b). With this motivation, we are now working on various follow-up observations in the multi-wavelength regime. Upcoming results from these surveys will help us to characterise these massive HAEs in the near future.

On the other hand, HAEs outside of the dense cores have similar SFR and stellar mass distributions to those in the random field (Sobral et al. 2013; Davidzon et al. 2017). These suggests that environmental impacts are not critical in intermediate densities in young protoclusters i.e. an early stage of inside-out growth of the galaxy clusters. This also means that high sampling density enough to resolve substructures on smaller than $\sim 1 \mathrm{Mpc}$ scale should be needed to identify early environmental effects as represented by our deep narrowband survey. If not, poor sampling density would lead us to misinterpret obtained results despite the existence of some important features hidden in the densest substructures in protoclusters.

The total SFR densities estimated from integration of the $\mathrm{H} \alpha$ luminosity function down to $L_{\mathrm{H} \alpha}=1 \times 10^{42} \mathrm{erg} \mathrm{s}^{-1}$ is $12 \mathrm{M}_{\odot} \mathrm{yr}^{-1} \mathrm{Mpc}^{-3}$ and $1111 \mathrm{M}_{\odot} \mathrm{yr}^{-1} \mathrm{Mpc}^{-3}$ in the intergroup and the group regions, corresponding to two and four orders of magnitude higher SFR volume densities than that in the general field (Madau et al. 1996; Lilly et al. 1996; Madau \& Dickinson 2014) at the same redshift, respectively. Such high cosmic SFR densities in dense cores of protoclusters will rapidly decline from $z \sim 2$ to the local Universe by the scale of $\propto(1+z)^{6-7}$ (Kodama et al. 2004; Finn et al. 2005; Cooper et al. 2008; Koyama et al. 2010, 2011; Hayashi et al. 2011; Webb et al. 2013; Smail et al. 2014; Clements et al. 2014; Shimakawa et al. 2014; Kato et al. 2016). This drastic decrease of star-formation densities is intimately related to a rapidly increasing fraction of quenching populations (Baldry et al. 2006; Peng et al. 2010b; Sobral et al. 2011; Muzzin et al. 2012; Darvish et al. 2016) driven perhaps by cold gas depletion (strangulation), energy injections of member galaxies and AGNs, and environmental quenching (e.g. Jaffé et al. 2015; Noble et al. 2016; Maier et al. 2016; Valentino et al. 2016; Wang et al. 2016; Hayashi et al. 2017b, and simulations by e.g. Romeo et al. 2005; Sijacki et al. 2007; Fabjan et al. 2010; Vogelsberger et al. 2014). In the meanwhile, star-forming activity propagates towards the outskirts and in smaller systems in the context of inside-out growth of the galaxy clusters (Poggianti et al. 2006; Elbaz et al. 2007; Koyama et al. 2011; van der Burg et al. 2015; Chiang et al. 2017). Eventually, the picture is entirely reversed in the sense that galaxies in massive clusters and groups tend to have lower SFRs than the general field in the present-day galaxy clusters and dense group environments as successfully represented by the Sloan Digital Sky Survey (Lewis et al. 2002; Gómez et al. 2003; Kauffmann et al. 2004, see also Alberts et al. 2014).

Moreover, the other intriguing feature discovered by this paper is the identification of enhanced SFRs across the main sequence in very high local densities. So far, while the past similar studies have found enhanced SFRs along the starforming main sequence due to the number excess of massive HAEs (Koyama et al. 2013a,b), these massive HAEs are shifted right upward on the $\mathrm{M}_{\star}-\mathrm{SFR}$ plane nearly alongside of the main sequence and do not show deviation from the sequence (see also Brodwin et al. 2013). In fact, we do not identify any significant difference of $\Delta \mathrm{MS}$ between the group and the intergroup HAEs, and only see a deviation when we focus on the top quartile of overdensities. This suggests that relatively large sample size enough to trace substructures may be important to identify the significant difference in such scattered empirical relationship. Combined with mass enhancement in the group regions, the densest regions in young protoclusters would have had accelerated and enhanced galaxy formation, which has been implied in the past studies for (proto)clusters of galaxies at lower redshifts (e.g. Steidel et al. 2005; Thomas et al. 2005; Strazzullo et al. 2010; Tanaka et al. 2013; Ferré-Mateu et al. 2014; Cerulo et al. 2016, but see Thomas et al. 2010; Gobat et al. 2013).

However, we have no clear explanation of physical origins of the enhanced star formation of HAEs in the extreme overdensities. We here propose two scenarios which may be able to examine the enhanced star formation of galaxies in the densest structures in the young protocluster: (1) galaxygalaxy interaction and (2) a vast amount of cold gas associated with the densest substructures, as described below.

(1) Because of very high densities no larger than $\bar{b}_{5 \text { th }}=$ $150 \mathrm{ph}-\mathrm{kpc}$ in the mean projected separations, it may be expected that there are lots of physical interactions among protocluster members therein. In high-density regions, galaxy collisions would occur more often as predicted theoretically (Okamoto \& Habe 2000; Gottlöber et al. 2001; Fakhouri \& Ma 2009; Jian et al. 2012), and also observationally (Lin et al. 2010; Kampczyk et al. 2013; Hine et al. 2016, but see Darg et al. 2010). This helps protocluster galaxies to grow into the compact, spheroidal galaxies seen in the local galaxy clusters through dissipational processes (Mihos \& Hernquist 1996; Barnes \& Hernquist 1996; Bournaud et al. 2007; Jesseit et al. 2009; Hopkins et al. 2008, 2010). Indeed, there are several merger-like systems in our HAE sample identifiable by visual inspection of F160W/WFC3 images from HST; however, it is hard to classify those as mergers or clumpy galaxies based only on rest-optical images. Follow-up integral field spectroscopy would constrain their kinematics. Furthermore, merger-induced starbursts are likely stochastic events and may not produce a systematic upward offset from the star-forming main sequence. Taking account these issues, we disfavour the primary explanation by galaxy-galaxy interactions.

(2) Circumgalactic/intergalactic media (CGM/IGM) are critical components for galaxy formation. This is crucially linked to the gas feeding mechanism of galaxies that depends on the cosmic age, halo mass and environment (Kereš et al. 2005; van de Voort et al. 2011). At high redshifts $(z \gtrsim 2)$, as reported by the recent hydrodynamic simulations, more efficient radiative cooling and self-shielding at high redshifts allow gas to cool and penetrate into galaxies in even hot massive halos larger than $1 \times 10^{12} \mathrm{M}_{\odot}$ since those still hold a significant amount of cold Hi gas (Dekel \& Birnboim 2006; Dekel et al. 2009a,b; Kereš et al. 2009; Faucher-Giguère et al. 2010; van de Voort et al. 2011, see also more classical picture in Gunn \& Gott 1972). The vast amount of Hi gas associated with the young protocluster cores might be able to examine $\operatorname{Ly} \alpha$ depletion effect and more extended diffuse Ly $\alpha$ haloes in overdensities (Matsuda 
et al. 2012; Hennawi et al. 2015; Shimakawa et al. 2017a), and Hi column density distribution at high redshifts (van de Voort et al. 2012). On a large scale, at least, recent observations have revealed that massive Hi structures are associated with protoclusters (Lee et al. 2016; Cai et al. 2017; Mawatari et al. 2017). Furthermore, cold accretion into the hot cores as known as the cold stream (Dekel et al. 2009a), not only help massive galaxies to maintain their star-forming activities but also could supply surrounding members sharing the dense group cores with sufficient cold gas reservoirs. This might involve more active star formation and then produce more dust in the entire HAEs in the densest groups. If true, such a vigorous gas feeding mechanism can systematically boost star formation of galaxies associated with the young protocluster's core. To test this hypothesis, we desire additional observational constraints from another aspect (e.g. $\mathrm{CO}$ observation of the protocluster centre; Emonts et al. 2016).

Finally, we acknowledge every result reported in this paper is based on only one dense protocluster system at $z=2.53$. A more comprehensive and systematic analyses is highly desirable to establish a consensus view on galaxy formation in the galaxy cluster. For instance, more than a thousand galaxies or ten dense protoclusters would be preferable to significantly improve the derivation of the stellar mass function in the protocluster region. On top of that, ideally, we need to investigate both star-forming and passive galaxies in galaxy clusters across cosmic time, and then build a selfconsistent model of quenching histories of cluster galaxies. On-going extensive programs such as MAMMOTH (MApping the Most Massive Overdensity Through Hydrogen, Cai et al. 2016) and the Subaru Strategic Program with Hyper Suprime-Cam (Aihara et al. 2017) have great potential to construct a large protocluster sample to address this problem in the near future.

\section{SUMMARY}

- Deep H $\alpha$ line imaging of the protocluster, USS 1558 at $z=2.53$ with MOIRCS on the Subaru Telescope has succeeded in discovering a total of 107 HAEs within just a $4^{\prime} \times 7^{\prime}$ square arcmin area. Stellar masses and dust-corrected SFRs are measured down to $1 \times 10^{8} \mathrm{M}_{\odot}$ and $3 \mathrm{M}_{\odot} \mathrm{yr}^{-1}$, respectively. The high sampling density by deep panoramic $\mathrm{H} \alpha$ mapping enables the analyses of early environmental effects of galaxy properties within the protocluster, on substructure scales smaller than one ph-Mpc. We derived a density peak with galaxies by the mean projected separation less than 100 ph-kpc scales. We then divide the HAEs into subsamples by overdensity to compare physical properties with local environment in the protocluster.

- This work compared physical properties of HAEs between lower and higher 50th percentile densities within the protocluster. We find enhancements of SFRs and dust reddening of HAEs in the dense group cores. At a given stellar mass, we only see statistical differences in the dust reddening between the group and the intergroup regions. However, we find that HAEs in the supergroup regions (top quartile overdensity) show significant SFR enhancements across the main sequence. Moreover, we have identified that $\mathrm{H} \alpha$ luminosity and stellar mass distributions are remarkably different between the group and intergroup environments in the sense that the dense group regions in the protocluster have significantly higher cutoff $\mathrm{H} \alpha$ luminosity and stellar mass than the intergroup regions. These results suggest that the densest environment in the early phase of inside-out growth of the galaxy clusters contribute greatly to the rapid building of very massive galaxies. Importantly, these unique features can be found when one has sufficiently deep data and high sampling density to detect faint objects and resolve substructures in the protocluster.

- We argue that the densest parts of the young protocluster take the lead in prompt and early construction of very massive galaxies. These will eventually grow into the brightend red sequence of objects commonly seen in present-day massive clusters of galaxies. Meanwhile, in the early forming protocluster phase, intergroup environments have little involvement in building such massive galaxies in accordance with the primary stage of inside-out evolution of galaxy clusters. Exploring the kinematics of merging protocluster members and focusing on gas feeding processes into protocluster's cores is crucial to reveal the physical phenomena that boost those star formation.

\section{ACKNOWLEDGEMENTS}

The data are collected at the Subaru Telescope, which is operated by the National Astronomical Observatory of Japan. A part of analyses is conducted with the assistance of the Tool for OPerations on Catalogues And Tables (торсAт; Taylor 2015). This work gains the benefit from the 3D-HST Treasury Program (GO 12177 and 12328) with NASA/ESA HST, which is operated by the Association of Universities for Research in Astronomy, Inc., under NASA contract NAS526555. We thank the anonymous referee for useful comments. R.S. acknowledge the support from the Japan Society for the Promotion of Science (JSPS) through JSPS overseas research fellowships. T.K. acknowledges KAKENHI No. 21340045.

\section{REFERENCES}

Aihara H., et al., 2017, preprint, (arXiv:1704.05858)

Alberts S., et al., 2014, MNRAS, 437, 437

An F. X., et al., 2014, ApJ, 784, 152

Ao Y., et al., 2017, preprint, (arXiv:1704.05101)

Atek H., et al., 2011, ApJ, 743, 121

Baldry I. K., Balogh M. L., Bower R. G., Glazebrook K., Nichol R. C., Bamford S. P., Budavari T., 2006, MNRAS, 373, 469

Balogh M. L., Christlein D., Zabludoff A. I., Zaritsky D., 2001, ApJ, 557, 117

Bamford S. P., et al., 2009, MNRAS, 393, 1324

Barnes J. E., Hernquist L., 1996, ApJ, 471, 115

Behroozi P. S., Wechsler R. H., Conroy C., 2013, ApJ, 770, 57

Belli S., Newman A. B., Ellis R. S., Konidaris N. P., 2014, ApJ, 788, L29

Bertin E., Arnouts S., 1996, A\&AS, 117, 393

Bezanson R., van Dokkum P. G., Tal T., Marchesini D., Kriek M., Franx M., Coppi P., 2009, ApJ, 697, 1290

Blanton M. R., Moustakas J., 2009, ARA\&A, 47, 159

Bolzonella M., et al., 2010, A\&A, 524, A76

Bournaud F., Jog C. J., Combes F., 2007, A\&A, 476, 1179 
Bournaud F., Daddi E., Weiß A., Renaud F., Mastropietro C., Teyssier R., 2015, A\&A, 575, A56

Bower R. G., Lucey J. R., Ellis R. S., 1992, MNRAS, 254, 601

Bower R. G., Kodama T., Terlevich A., 1998, MNRAS, 299, 1193

Brammer G. B., et al., 2012, ApJS, 200, 13

Brinchmann J., Charlot S., White S. D. M., Tremonti C., Kauffmann G., Heckman T., Brinkmann J., 2004, MNRAS, 351, 1151

Brodwin M., et al., 2013, ApJ, 779, 138

Brough S., et al., 2017, ApJ, 844, 59

Bruzual G., Charlot S., 2003, MNRAS, 344, 1000

Butcher H., Oemler Jr. A., 1984, ApJ, 285, 426

Cai Z., et al., 2016, ApJ, 833, 135

Cai Z., et al., 2017, ApJ, 839, 131

Calzetti D., Armus L., Bohlin R. C., Kinney A. L., Koornneef J., Storchi-Bergmann T., 2000, ApJ, 533, 682

Cappellari M., et al., 2011, MNRAS, 416, 1680

Cerulo P., et al., 2016, MNRAS, 457, 2209

Chabrier G., 2003, PASP, 115, 763

Chiang Y.-K., Overzier R., Gebhardt K., 2013, ApJ, 779, 127

Chiang Y.-K., Overzier R. A., Gebhardt K., Henriques B., 2017, ApJ, 844, L23

Clements D. L., et al., 2014, MNRAS, 439, 1193

Cochrane R. K., Best P. N., Sobral D., Smail I., Wake D. A., Stott J. P., Geach J. E., 2017, MNRAS, 469, 2913

Conselice C. J., 2002, ApJ, 573, L5

Cooke E. A., Hatch N. A., Muldrew S. I., Rigby E. E., Kurk J. D., 2014, MNRAS, 440, 3262

Cooper M. C., et al., 2008, MNRAS, 383, 1058

Couch W. J., Barger A. J., Smail I., Ellis R. S., Sharples R. M., 1998, ApJ, 497, 188

Daddi E., Cimatti A., Renzini A., Fontana A., Mignoli M., Pozzetti L., Tozzi P., Zamorani G., 2004, ApJ, 617, 746

Daddi E., et al., 2007, ApJ, 670, 156

Dannerbauer H., et al., 2014, A\&A, 570, A55

Darg D. W., et al., 2010, MNRAS, 401, 1552

Darvish B., Mobasher B., Sobral D., Rettura A., Scoville N., Faisst A., Capak P., 2016, ApJ, 825, 113

Davidzon I., et al., 2017, preprint, (arXiv:1701.02734)

De Propris R., et al., 2003, MNRAS, 342, 725

Dekel A., Birnboim Y., 2006, MNRAS, 368, 2

Dekel A., et al., 2009a, Nature, 457, 451

Dekel A., Sari R., Ceverino D., 2009b, ApJ, 703, 785

Dressler A., 1980, ApJ, 236, 351

Dressler A., et al., 1997, ApJ, 490, 577

Elbaz D., et al., 2007, A\&A, 468, 33

Emonts B. H. C., et al., 2016, Science, 354, 1128

Erb D. K., Bogosavljević M., Steidel C. C., 2011, ApJ, 740, L31

Fabjan D., Borgani S., Tornatore L., Saro A., Murante G., Dolag K., 2010, MNRAS, 401, 1670

Fakhouri O., Ma C.-P., 2009, MNRAS, 394, 1825

Faucher-Giguère C.-A., Kereš D., Dijkstra M., Hernquist L., Zaldarriaga M., 2010, ApJ, 725, 633

Ferré-Mateu A., Sánchez-Blázquez P., Vazdekis A., de la Rosa I. G., 2014, ApJ, 797, 136

Finn R. A., et al., 2005, ApJ, 630, 206

Fitzpatrick E. L., 1999, PASP, 111, 63

Fogarty L. M. R., et al., 2014, MNRAS, 443, 485

Franx M., et al., 2003, ApJ, 587, L79

Fumagalli M., O'Meara J. M., Prochaska J. X., Kanekar N., 2010, MNRAS, 408, 362

Gabor J. M., Davé R., 2012, MNRAS, 427, 1816

Galametz A., et al., 2012, ApJ, 749, 169

Garn T., Best P. N., 2010, MNRAS, 409, 421

Genel S., 2016, ApJ, 822, 107

Genel S., et al., 2014, MNRAS, 445, 175

Gladders M. D., Yee H. K. C., 2000, AJ, 120, 2148

Gobat R., et al., 2011, A\&A, 526, A133
Gobat R., et al., 2013, ApJ, 776, 9

Gobat R., et al., 2015, A\&A, 581, A56

Gómez P. L., et al., 2003, ApJ, 584, 210

Goto T., et al., 2003, PASJ, 55, 739

Gottlöber S., Klypin A., Kravtsov A. V., 2001, ApJ, 546, 223

Gunn J. E., Gott III J. R., 1972, ApJ, 176, 1

Hatch N. A., Kurk J. D., Pentericci L., Venemans B. P., Kuiper E., Miley G. K., Röttgering H. J. A., 2011, MNRAS, 415, 2993

Hatch N. A., Cooke E. A., Muldrew S. I., Hartley W. G., Almaini O., Conselice C. J., Simpson C. J., 2017, MNRAS, 464, 876

Hayashi M., Kodama T., Koyama Y., Tadaki K.-I., Tanaka I., 2011, MNRAS, 415, 2670

Hayashi M., Kodama T., Tadaki K.-i., Koyama Y., Tanaka I., 2012, ApJ, 757, 15

Hayashi M., Kodama T., Tanaka I., Shimakawa R., Koyama Y., Tadaki K.-i., Suzuki T. L., Yamamoto M., 2016, ApJ, 826, L28

Hayashi M., et al., 2017a, preprint, (arXiv:1704.05978)

Hayashi M., et al., 2017b, ApJ, 841, L21

Hennawi J. F., Prochaska J. X., Cantalupo S., Arrigoni-Battaia F., 2015, Science, 348, 779

Hine N. K., Geach J. E., Alexander D. M., Lehmer B. D., Chapman S. C., Matsuda Y., 2016, MNRAS, 455, 2363

Hopkins P. F., Hernquist L., Cox T. J., Kereš D., 2008, ApJS, 175,356

Hopkins P. F., Bundy K., Hernquist L., Wuyts S., Cox T. J., 2010, MNRAS, 401, 1099

Houghton R. C. W., et al., 2013, MNRAS, 436, 19

Husband K., Bremer M. N., Stott J. P., Murphy D. N. A., 2016, MNRAS, 462, 421

Ibar E., et al., 2013, MNRAS, 434, 3218

Ichikawa T., et al., 2006, in Society of Photo-Optical Instrumentation Engineers (SPIE) Conference Series. , doi: $10.1117 / 12.670078$

Ilbert O., et al., 2013, A\&A, 556, A55

Jaffé Y. L., Smith R., Candlish G. N., Poggianti B. M., Sheen Y.-K., Verheijen M. A. W., 2015, MNRAS, 448, 1715

Jesseit R., Cappellari M., Naab T., Emsellem E., Burkert A., 2009, MNRAS, 397, 1202

Jian H.-Y., Lin L., Chiueh T., 2012, ApJ, 754, 26

Jiménez N., Cora S. A., Bassino L. P., Tecce T. E., Smith Castelli A. V., 2011, MNRAS, 417, 785

Kacprzak G. G., et al., 2015, ApJ, 802, L26

Kajisawa M., Kodama T., Tanaka I., Yamada T., Bower R., 2006, MNRAS, 371, 577

Kampczyk P., et al., 2013, ApJ, 762, 43

Kato Y., et al., 2016, MNRAS, 460, 3861

Kauffmann G., White S. D. M., Heckman T. M., Ménard B., Brinchmann J., Charlot S., Tremonti C., Brinkmann J., 2004, MNRAS, 353, 713

Kelkar K., Gray M. E., Aragón-Salamanca A., Rudnick G., Milvang-Jensen B., Jablonka P., Schrabback T., 2017, MNRAS, 469, 4551

Kennicutt Jr. R. C., 1998, ARA\&A, 36, 189

Kereš D., Katz N., Weinberg D. H., Davé R., 2005, MNRAS, 363, 2

Kereš D., Katz N., Davé R., Fardal M., Weinberg D. H., 2009, MNRAS, 396, 2332

Knopp G. P., Chambers K. C., 1997, ApJS, 109, 367

Kodama T., Balogh M. L., Smail I., Bower R. G., Nakata F., 2004, MNRAS, 354, 1103

Kodama T., Tanaka I., Kajisawa M., Kurk J., Venemans B., De Breuck C., Vernet J., Lidman C., 2007, MNRAS, 377, 1717

Kodama T., Hayashi M., Koyama Y., Tadaki K.-i., Tanaka I., Shimakawa R., 2013, in Thomas D., Pasquali A., Ferreras I., eds, IAU Symposium Vol. 295, IAU Symposium. pp 74-77, doi:10.1017/S1743921313004353

Koester B. P., et al., 2007, ApJ, 660, 239 
Koyama Y., Kodama T., Shimasaku K., Hayashi M., Okamura S., Tanaka I., Tokoku C., 2010, MNRAS, 403, 1611

Koyama Y., Kodama T., Nakata F., Shimasaku K., Okamura S., 2011, ApJ, 734, 66

Koyama Y., Kodama T., Tadaki K.-i., Hayashi M., Tanaka M., Smail I., Tanaka I., Kurk J., 2013a, MNRAS, 428, 1551

Koyama Y., et al., 2013b, MNRAS, 434, 423

Koyama Y., Kodama T., Tadaki K.-i., Hayashi M., Tanaka I., Shimakawa R., 2014, ApJ, 789, 18

Koyama Y., et al., 2015, MNRAS, 453, 879

Kriek M., van Dokkum P. G., Labbé I., Franx M., Illingworth G. D., Marchesini D., Quadri R. F., 2009, ApJ, 700, 221

Kriek M., et al., 2015, ApJS, 218, 15

Kubo M., Yamada T., Ichikawa T., Kajisawa M., Matsuda Y., Tanaka I., Umehata H., 2017, MNRAS, 469, 2235

Kulas K. R., et al., 2013, ApJ, 774, 130

Kurk J. D., et al., 2000, A\&A, 358, L1

Kurk J. D., Pentericci L., Röttgering H. J. A., Miley G. K., 2004, A\&A, 428, 793

Lani C., et al., 2013, MNRAS, 435, 207

Lee K.-G., et al., 2014, ApJ, 795, L12

Lee K.-G., et al., 2016, ApJ, 817, 160

Lehmer B. D., et al., 2009, ApJ, 691, 687

Lehmer B. D., et al., 2013, ApJ, 765, 87

Lewis I., et al., 2002, MNRAS, 334, 673

Lilly S. J., Le Fevre O., Hammer F., Crampton D., 1996, ApJ, 460, L1

Lin L., et al., 2010, ApJ, 718, 1158

Lin L., et al., 2012, ApJ, 756, 71

Lintott C. J., et al., 2008, MNRAS, 389, 1179

Lintott C., et al., 2011, MNRAS, 410, 166

Madau P., Dickinson M., 2014, ARA\&A, 52, 415

Madau P., Ferguson H. C., Dickinson M. E., Giavalisco M., Steidel C. C., Fruchter A., 1996, MNRAS, 283, 1388

Maier C., et al., 2016, A\&A, 590, A108

Markwardt C. B., 2009, in Bohlender D. A., Durand D., Dowler P., eds, Astronomical Society of the Pacific Conference Series Vol. 411, Astronomical Data Analysis Software and Systems XVIII. p. 251 (arXiv:0902.2850)

Matsuda Y., et al., 2011a, MNRAS, 410, L13

Matsuda Y., et al., 2011b, MNRAS, 416, 2041

Matsuda Y., et al., 2012, MNRAS, 425, 878

Matthee J., Sobral D., Best P., Smail I., Bian F., Darvish B., Röttgering H., Fan X., 2017, MNRAS, 471, 629

Mawatari K., et al., 2017, MNRAS, 467, 3951

Mayo J. H., Vernet J., De Breuck C., Galametz A., Seymour N., Stern D., 2012, A\&A, 539, A33

Mei S., et al., 2009, ApJ, 690, 42

Mihos J. C., Hernquist L., 1996, ApJ, 464, 641

Miyazaki S., et al., 2002, PASJ, 54, 833

Mortlock A., et al., 2015, MNRAS, 447, 2

Muldrew S. I., Hatch N. A., Cooke E. A., 2015, MNRAS, 452, 2528

Muzzin A., et al., 2012, ApJ, 746, 188

Muzzin A., et al., 2013, ApJ, 777, 18

Naab T., Johansson P. H., Ostriker J. P., 2009, ApJ, 699, L178

Newman A. B., Ellis R. S., Andreon S., Treu T., Raichoor A., Trinchieri G., 2014, ApJ, 788, 51

Noble A. G., Webb T. M. A., Yee H. K. C., Muzzin A., Wilson G., van der Burg R. F. J., Balogh M. L., Shupe D. L., 2016, ApJ, 816, 48

Noeske K. G., et al., 2007, ApJ, 660, L43

Okamoto T., Habe A., 2000, PASJ, 52, 457

Oke J. B., Gunn J. E., 1983, ApJ, 266, 713

Oteo I., Sobral D., Ivison R. J., Smail I., Best P. N., Cepa J., Pérez-García A. M., 2015, MNRAS, 452, 2018

Oteo I., et al., 2017, preprint, (arXiv:1709.02809)

Papovich C., et al., 2012, ApJ, 750, 93
Peebles P. J. E., 1970, AJ, 75, 13

Peng C. Y., Ho L. C., Impey C. D., Rix H.-W., 2010a, AJ, 139, 2097

Peng Y.-j., et al., 2010b, ApJ, 721, 193

Penny S. J., Conselice C. J., 2008, MNRAS, 383, 247

Planck Collaboration et al., 2015, A\&A, 582, A30

Poggianti B. M., et al., 2006, ApJ, 642, 188

Price S. H., et al., 2014, ApJ, 788, 86

Rakic O., Schaye J., Steidel C. C., Rudie G. C., 2012, ApJ, 751, 94

Reddy N. A., Erb D. K., Pettini M., Steidel C. C., Shapley A. E., 2010, ApJ, 712, 1070

Reddy N. A., et al., 2015, ApJ, 806, 259

Romeo A. D., Portinari L., Sommer-Larsen J., 2005, MNRAS, 361,983

Salmon B., et al., 2015, ApJ, 799, 183

Sanders R. L., et al., 2015, ApJ, 799, 138

Schaerer D., de Barros S., Sklias P., 2013, A\&A, 549, A4

Schechter P., 1976, ApJ, 203, 297

Schlafly E. F., Finkbeiner D. P., 2011, ApJ, 737, 103

Schlegel D. J., Finkbeiner D. P., Davis M., 1998, ApJ, 500, 525

Shen S., Mo H. J., White S. D. M., Blanton M. R., Kauffmann G., Voges W., Brinkmann J., Csabai I., 2003, MNRAS, 343, 978

Shimakawa R., Kodama T., Tadaki K.-i., Tanaka I., Hayashi M., Koyama Y., 2014, MNRAS, 441, L1

Shimakawa R., Kodama T., Tadaki K.-i., Hayashi M., Koyama Y., Tanaka I., 2015a, MNRAS, 448, 666

Shimakawa R., et al., 2015b, MNRAS, 451, 1284

Shimakawa R., et al., 2017a, MNRAS, 468, L21

Shimakawa R., et al., 2017b, MNRAS, 468, 1123

Shivaei I., et al., 2015, ApJ, 815, 98

Sijacki D., Springel V., Di Matteo T., Hernquist L., 2007, MNRAS, 380, 877

Skelton R. E., et al., 2014, ApJS, 214, 24

Skibba R. A., et al., 2009, MNRAS, 399, 966

Smail I., et al., 2014, ApJ, 782, 19

Snyder G. F., et al., 2012, ApJ, 756, 114

Sobral D., et al., 2009, MNRAS, 398, 75

Sobral D., Best P. N., Smail I., Geach J. E., Cirasuolo M., Garn T., Dalton G. B., 2011, MNRAS, 411, 675

Sobral D., Best P. N., Matsuda Y., Smail I., Geach J. E., Cirasuolo M., 2012, MNRAS, 420, 1926

Sobral D., Smail I., Best P. N., Geach J. E., Matsuda Y., Stott J. P., Cirasuolo M., Kurk J., 2013, MNRAS, 428, 1128

Sobral D., Best P. N., Smail I., Mobasher B., Stott J., Nisbet D., 2014, MNRAS, 437, 3516

Sobral D., Kohn S. A., Best P. N., Smail I., Harrison C. M., Stott J., Calhau J., Matthee J., 2016, MNRAS, 457, 1739

Speagle J. S., Steinhardt C. L., Capak P. L., Silverman J. D., 2014, ApJS, 214, 15

Springel V., et al., 2005, Nature, 435, 629

Stark D. P., Schenker M. A., Ellis R., Robertson B., McLure R., Dunlop J., 2013, ApJ, 763, 129

Steidel C. C., Adelberger K. L., Dickinson M., Giavalisco M., Pettini M., Kellogg M., 1998, ApJ, 492, 428

Steidel C. C., Adelberger K. L., Shapley A. E., Erb D. K., Reddy N. A., Pettini M., 2005, ApJ, 626, 44

Steidel C. C., et al., 2014, ApJ, 795, 165

Stott J. P., Pimbblet K. A., Edge A. C., Smith G. P., Wardlow J. L., 2009, MNRAS, 394, 2098

Stott J. P., Sobral D., Smail I., Bower R., Best P. N., Geach J. E., 2013, MNRAS, 430, 1158

Strazzullo V., et al., 2010, A\&A, 524, A17

Strazzullo V., et al., 2013, ApJ, 772, 118

Strazzullo V., et al., 2016, ApJ, 833, L20

Sunyaev R. A., Zeldovich Y. B., 1972, A\&A, 20, 189

Suzuki R., et al., 2008, PASJ, 60, 1347 
Tadaki K.-i., et al., 2017, ApJ, 834, 135

Tanaka M., Kodama T., Arimoto N., Okamura S., Umetsu K., Shimasaku K., Tanaka I., Yamada T., 2005, MNRAS, 362, 268

Tanaka I., et al., 2011, PASJ, 63, 415

Tanaka M., et al., 2013, PASJ, 65, 17

Taylor M. B., 2015, in Taylor A. R., Rosolowsky E., eds, Astronomical Society of the Pacific Conference Series Vol. 495, Astronomical Society of the Pacific Conference Series. p. 177 (arXiv: 1410.8507)

Terlevich A. I., Caldwell N., Bower R. G., 2001, MNRAS, 326, 1547

Teyssier R., Chapon D., Bournaud F., 2010, ApJ, 720, L149

Thomas D., Maraston C., Bender R., Mendes de Oliveira C., 2005, ApJ, 621, 673

Thomas D., Maraston C., Schawinski K., Sarzi M., Silk J., 2010, MNRAS, 404, 1775

Tomczak A. R., et al., 2016, ApJ, 817, 118

Tomczak A. R., et al., 2017, preprint, (arXiv:1709.00011)

Toshikawa J., et al., 2016, ApJ, 826, 114

Toshikawa J., et al., 2017, preprint, (arXiv:1708.09421)

Tran K.-V. H., et al., 2015, ApJ, 811, 28

Tran K.-V. H., et al., 2017, ApJ, 834, 101

Trentham N., 1998, MNRAS, 294, 193

Valentino F., et al., 2015, ApJ, 801, 132

Valentino F., et al., 2016, ApJ, 829, 53

Venemans B. P., et al., 2002, ApJ, 569, L11

Vilella-Rojo G., et al., 2015, A\&A, 580, A47

Visvanathan N., Sandage A., 1977, ApJ, 216, 214

Vogelsberger M., et al., 2014, MNRAS, 444, 1518

Wang T., et al., 2016, ApJ, 828, 56

Webb T. M. A., et al., 2013, AJ, 146, 84

Wetzel A. R., Tinker J. L., Conroy C., van den Bosch F. C., 2013, MNRAS, 432, 336

Whitaker K. E., van Dokkum P. G., Brammer G., Franx M., 2012, ApJ, 754, L29

Whitaker K. E., et al., 2014, ApJ, 795, 104

Wolfe A. M., Gawiser E., Prochaska J. X., 2005, ARA\&A, 43, 861

Wuyts S., et al., 2007, ApJ, 655, 51

Wuyts S., et al., 2012, ApJ, 753, 114

Yagi M., Kashikawa N., Sekiguchi M., Doi M., Yasuda N., Shimasaku K., Okamura S., 2002, AJ, 123, 66

Zahid H. J., Yates R. M., Kewley L. J., Kudritzki R. P., 2013, ApJ, 763, 92

Zehavi I., et al., 2011, ApJ, 736, 59

Zirm A. W., et al., 2008, ApJ, 680, 224

de Vaucouleurs G., 1961, ApJS, 5, 233

van Albada G. B., 1961, AJ, 66, 590

van Dokkum P. G., et al., 2010, ApJ, 709, 1018

van de Voort F., Schaye J., Booth C. M., Haas M. R., Dalla Vecchia C., 2011, MNRAS, 414, 2458

van de Voort F., Schaye J., Altay G., Theuns T., 2012, MNRAS, 421, 2809

van der Burg R. F. J., Hoekstra H., Muzzin A., Sifón C., Balogh M. L., McGee S. L., 2015, A\&A, 577, A19

van der Wel A., et al., 2012, ApJS, 203, 24

van der Wel A., et al., 2014, ApJ, 788, 28

\section{APPENDIX A: CATALOGUE \\ APPENDIX B: COMPLETENESS}

We calculate completeness as a function of narrowband magnitude by using a Monte Carlo simulation. First, we embedded 10 PSF objects at the narrowband magnitude of 19, 20, and from 21 to $25 \mathrm{mag}$ in step of $0.2 \mathrm{mag}$ to the reduced

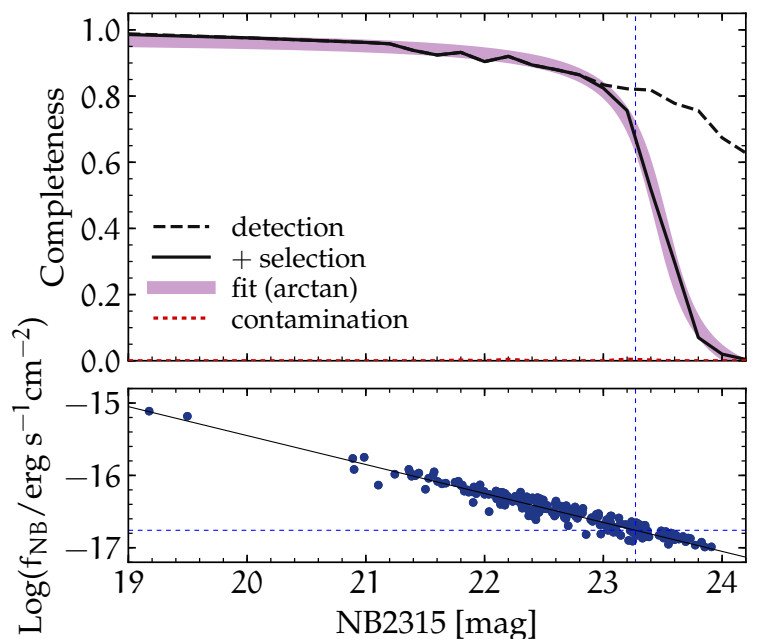

Figure B1. Dashed line presents the detection completeness as a function of narrowband magnitude, and the solid line includes the selection completeness. We use an arctangent function as fitted to the black line by the purple thick curve. The red dotted line indicates the contamination rate from photometric errors. In the lower figure, blue circles show log narrowband fluxes $\left(f_{N B}\right)$ of our narrowband emitters. The black solid line indicates the fit to the observed emitter flux for the Monte Carlo simulation on selection completeness. The blue vertical and perpendicular lines indicate 68 per cent completeness, as estimated from our simulation.

NB2315 image, and then test the recovery rate i.e. detection completeness of sources detected by the SExtractor (version 2.19.5; Bertin \& Arnouts 1996). This test was repeated 50 times, and thus a total 500 PSF of objects were computed for a given magnitude. The derived detection completeness is represented in Fig. B1.

We then demonstrate the recovery rate by the narrowband selection expressed by the two equations 1 and 2 . This called selection completeness (Sobral et al. 2009, 2012, 2013) provides us with number of objects missed in the narrowband selection due to the colour-term variation and photometric errors of the $N B 2315$ and $K_{S}$ bands. Addressing the selection completeness is crucial to determine the completeness correction of the narrowband-selected emitter sample since the narrowband selection depends heavily on both photometric errors of narrowband (NB2315) and the counterpart broadband $\left(K_{S}\right)$ as described in the equation 1 and 2 .

Prior to the simulation, we estimated colour term variations of $K_{S}-N B 2315$ due to the difference of filter wavelength to introduce the intrinsic colour term errors for the simulation. Derivation of the typical colour term is important also to minimise the systematic error of derived narrowband flux and EW for our sample. Figure B2 shows colour term distributions across redshift between 0 and 4 inferred from spectral energy distributions (SED) of single stellar population (SSP) synthesis models (Bruzual \& Charlot 2003) with ages of $0.1,0.9$, and 2.5 Gyrs. These stellar metallicities are fixed to $Z=0.008$ with two different extinctions $\left(A_{V}=0\right.$ and 1 ). Also, the figure shows the colour terms of photometric redshift sources from 3D-HST (Skelton et al. 2014) whose SED spectra are based on the FAST SED-fitting code (Kriek et al. 2009). The initial parameters in the SED-fitting are the same as for the HAE samples when we estimate 
Table A1. Data catalogue of the HAE sample in USS 1558. Full table is available as online material.

\begin{tabular}{lccccccc}
\hline ID & $z_{\text {spec }}$ & $\begin{array}{c}\bar{b}_{\text {5th }} \\
(\mathrm{ph}-\mathrm{kpc})\end{array}$ & $\begin{array}{c}\log \left(\mathrm{M}_{\star}\right) \\
\left(\mathrm{M}_{\odot}\right)\end{array}$ & $\begin{array}{c}\mathrm{E}(\mathrm{B}-\mathrm{V}) \\
(\mathrm{mag})\end{array}$ & $\begin{array}{c}\log \left(\mathrm{SFR}_{\mathrm{H} \alpha}\right) \\
\left(\mathrm{M}_{\odot} \mathrm{yr}^{-1}\right)\end{array}$ & $\begin{array}{c}\log \left(\mathrm{R}_{e}\right) \\
(\mathrm{kpc})\end{array}$ & $\begin{array}{c}\mathrm{J}-\mathrm{Ks} \\
(\mathrm{mag})\end{array}$ \\
\hline 0002 & - & 292 & $8.76_{-0.13}^{+0.53}$ & $0.16_{-0.10}^{+0.03}$ & $0.72_{-0.19}^{+0.15}$ & - & $0.22 \pm 0.54$ \\
0003 & $-L$ & 415 & $8.08_{-0.28}^{+1.38}$ & $0.08_{-0.08}^{+0.07}$ & $0.77_{-0.16}^{+0.15}$ & - & - \\
0005 & - & 737 & $9.73_{-0.03}^{+0.04}$ & $0.23_{-0.01}^{+0.01}$ & $1.25_{-0.10}^{+0.10}$ & - & $-0.37 \pm 0.12$ \\
$\cdots$ & $\cdots$ & $\cdots$ & $\cdots$ & $\cdots$ & $\cdots$ & $\cdots$ & $\cdots$ \\
\hline
\end{tabular}

${ }^{L}$ Dual $\mathrm{H} \alpha$ and $\operatorname{Ly} \alpha$ emitters identified by Shimakawa et al. (2017a).

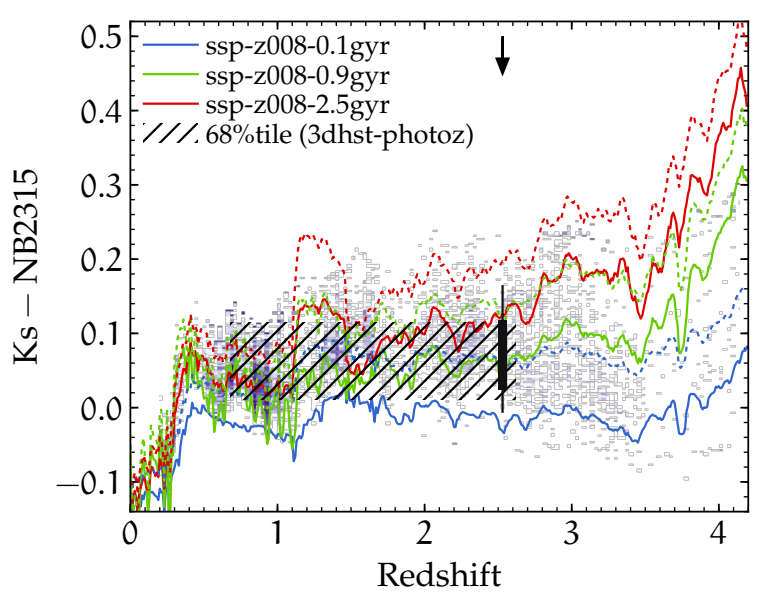

Figure B2. Colour term distributions of $K_{S}-N B 2315$ in SSP models (Bruzual \& Charlot 2003) with various ages (from the bottom, 0.1 Gyr: blue, 0.9 Gyr: green, and 2.5 Gyr: red). Solid and dashed curves assume zero extinction and $A_{V}=1$, respectively. The metallicities are fixed to $Z=0.008$. The black arrow indicates the narrowband redshift for $\mathrm{H} \alpha$ line $(z=2.53)$. The black hatch region indicates the 68 th percentile (\%tile) colour term distribution inferred from the SED of the photo- $z$ sources at $K_{S}=21-24$ mag from the 3D-HST (Skelton et al. 2014). Black thick and thin vertical lines indicate 68 th $\%$ and 95 th \%tiles of the colour-term distributions of HAEs inferred from the derived SEDs by the FAST SED fitting $(\S 3.2)$.

their physical properties ( $\$ 3.2)$. We also checked the colour term distribution with other models with different star formation histories or initial parameters for the SED-fitting, and confirmed that those colour terms do not greatly deviate from the range covered by those shown in the figure. We should note that none of these models include nebular emission components. We incorporate the colour term variation of $0.06 \pm 0.005$ inferred from photo- $z$ sources at $K_{s}=21-24$ mag (Fig. B2) in our simulation for the colour completeness, which is broadly consistent with that of derived HAE sample (Fig. B2).

We then conduct a Monte Carlo simulation similarly to the detection completeness, but we embed the PSF into the $K_{S}$ band image as well as NB2315 image. We allow for random variation in the $K_{S}$ magnitudes following the colour term distribution noted above. Moreover, we incorporate additional line flux to each PSF model for the narrowband image. We embedded a $\mathrm{H} \alpha$ line flux from the narrowband magnitude using the fitted power-law regression (shown in the lower panel of Fig. B1). Since narrowband magnitudes and derived narrowband fluxes of narrowband emitters are tightly correlated, this assumption would be reasonable. However, we should note that narrowband emitters with lower line fluxes should have lower completeness for a given narrowband magnitude. This could cause a systematic error for the analyses of distribution functions (§3.4) at the faint end. Based on recovered $N B$ and $K_{S}$-band photometry derived by the double image mode of the SExtractor, we calculate the recovery rate by counting objects which satisfy the narrowband selection criteria (eq. 1 and 2).

The final recovery rate combining the detection completeness with the selection completeness is shown in Fig. B1. We fit the result by an arctangent function,

$C(N B)=0.426-0.357 \times \operatorname{Arctan}(4.047 \times(N B-23.484))$,

which gives a better solution than polynomial and Gauss error functions. $C(N B)$ expresses the completeness value as a function of the narrowband magnitude of the HAE samples. We use this correction function to derive the $\mathrm{H} \alpha$ luminosity and stellar mass functions in $\S 3.4$. The 68 per cent completeness corresponds to a line flux of $1.7 \times 10^{-17} \mathrm{erg} \mathrm{s}^{-1} \mathrm{~cm}^{-2}$ or SFR of $4.2 \mathrm{M}_{\odot} \mathrm{yr}^{-1}$ at $z=2.53$.

We can also estimate the contamination rate due to photometric errors by using this simulation. We run the same simulation without adding line flux to the narrowband images, and then calculate the contaminants that show the $K_{S}-N B 2315$ colour excess due to those photometric errors including the colour term variation. As a result, we find that our selection criteria (eq. 1 and 2) effectively ignore such contaminations since the derived contamination rates are less than 0.2 per cent.

\section{APPENDIX C: EFFECT OF FILTER PROFILE}

Since our target is the protocluster at $z=2.53$, associated members are expected to gather around the centre of the system along the line of sight rather than homogeneously distribute within the redshift slice covered by the narrowband filter. Indeed, bright HAEs which have been confirmed by our past spectroscopy at NIR (Shimakawa et al. 2014, 2015b) tend to be located around the protocluster redshift (see literature or Fig. C2). In this context, the profile of the narrowband filter function should have a relatively smaller impact on the measured narrowband fluxes and the survey volume as compared to the narrowband imaging for line emitters in the general field.

However, we should carefully consider the obtained consequences by taking account of luminosity dependence on 
the clustering properties of HAEs as well, which has a potential to cause non-ignorable systematic errors in those measurements. Cochrane et al. (2017) have shown that clustering of HAEs should depend on $\mathrm{H} \alpha$ luminosity in the sense that more luminous HAEs preferentially reside in higher dark matter densities. Our dense protocluster sample may be an extreme case of such a tight connection between the clustering and $\mathrm{H} \alpha$ luminosity, as this work argues that bursty HAEs apt to be localised in the dense protocluster's cores. Thus, we should discuss the possibility that the intergroup members might be more widespread and thus more HAEs are located near the cut-on and cut-off of the filter response functions than HAEs in the dense group cores. Since such a systematic error may affect our results and conclusion, if any, what follows are a couple of simulations that test the effects by the narrowband filter profile on the derivation of $\mathrm{H} \alpha$ luminosity function and narrowband fluxes of HAEs.

\section{C1 Impacts on $\mathrm{H} \alpha$ luminosity function}

The partially top-hat function of the NB2315 narrowband filter essentially probes brighter HAEs in wider volume along the line of sight while those at the edge of the filter transmission curve can be selected as fainter sources due to the flux loss. As a result, observed $\mathrm{H} \alpha$ luminosity function can be steeper than the actual one since we would tend to unexpectedly count more/less HAEs in the fainter/brighter $\mathrm{H} \alpha$ luminosity range (Sobral et al. 2009). This could also produce the lower cutoff $\mathrm{H} \alpha$ luminosity in the intergroup regions than that of the dense groups as identified by this paper.

We simulate the impact of this issue by assuming that the intergroup HAEs distribute homogeneously along the line of sight. Although this assumption seems to be drastic according to the redshift distribution of the existing spec$z$ sources, it should be reasonable to evaluate the effect of the filter profile in such a worst possible case. The simulation is made with reference to Sobral et al. (2009). We first make more than $10^{5}$ samples with various $\mathrm{H} \alpha$ luminosities along the $\mathrm{H} \alpha$ luminosity function in the group region. We then spread the objects to random redshifts in the range of (C) $z=2.51-2.535$ or (F) $z=2.49-2.56$. The former redshift range roughly corresponds to the redshift distribution of HAEs the dense groups by Shimakawa et al. (2014), and the latter assumes that HAEs are distributed evenly over the $\mathrm{H} \alpha$ redshift covered by the narrowband filter. After that, we compute the recovered $\mathrm{H} \alpha$ luminosities depending on those redshifts according to the system throughput of the NB2315 filter. Based on the recovered $\mathrm{H} \alpha$ luminosities of the sample, we can reconstruct the $\mathrm{H} \alpha$ luminosity functions in the two cases. One should note that we here ignore the spatial dependence of filter response functions within the MOIRCS field of view (Tanaka et al. 2011). Furthermore, while we incorporate atmospheric transmission into the filter throughput, the filter profile still would not be demonstrated perfectly due to its dependence on such as temperature and angle of incidence.

Figure $\mathrm{C} 1$ shows the obtained retributed $\mathrm{H} \alpha$ luminosity functions for the group HAEs. Since the case (C) assumes the redshift distribution in the wavelength range where the narrowband filter is close to the top-hat function, the recovered functions exactly reproduces the original form. Also,

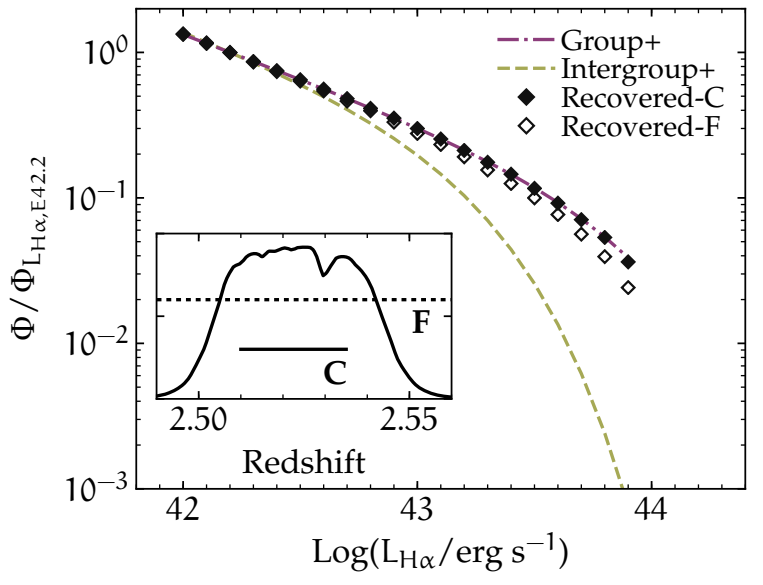

Figure C1. H $\alpha$ luminosity functions scaled at $L_{\mathrm{H} \alpha}=10^{42.2}$ erg $\mathrm{s}^{-1}$ (same as the left bottom panel in Fig. 9). The purple dash-dot and yellow dashed curves show the scaled $\mathrm{H} \alpha$ luminosity functions in the group and the intergroup regions. Black filled and open squares indicate recovered distribution functions for the group HAEs by retributing the sample into the different redshift space, at $z=2.51-2.535$ (pattern C) and 2.49-2.56 (pattern F), respectively (see the inset left below).

the result claims that the non-top-hat filter profile has only a minor effect on the $\mathrm{H} \alpha$ luminosity function, and more homogeneous redshift distributions of HAEs (F) cannot explain the clear gap of $\mathrm{H} \alpha$ luminosity functions between different densities at the bright end as seen in Fig. 9.

\section{C2 Impacts on the star-forming main sequence}

The past spectroscopic follow-up observations (Shimakawa et al. 2014, 2015b) do not cover the fainter HAE sources which are newly discovered by our deep narrowband imaging reported by this series of papers. Thus, we have few spectroscopically confirmed HAEs which appear below the main sequence. In fact, Figure C2 indicates that out past NIR spectroscopy mostly follows active HAEs, prevent us from investigating the difference of SFR distributions of HAEs in various local overdensities. In Fig. C2, we correct those flux loss due to the filter transmittance, which includes the correction of spatial dependence of the filter centre wavelength. Except for four HAEs at the filter edges, the measurement errors due to the filter profile for HAEs are smaller than 0.1 dex, suggesting that the flux loss in the narrowband filter would be a minor effect on our results and conclusions.

Besides, we carry out another simulation to check this issue even more carefully. If a larger number of HAEs in the intergroup regions were affected by the significant $\mathrm{H} \alpha$ flux loss relative to those in the dense group regions, it might lead to the relatively lower SFR estimate selectively for galaxies in intergroup regions (as seen in Fig. 6). To test such a possibility, we retribute observed narrowband flux distribution of the supergroup HAEs and then compare them with those in the intergroup HAEs by assuming the different redshift distribution as conducted in Appendix C1.

Figure C3 demonstrates how more widespread redshift distribution affects the narrowband flux distribution. We employ only HAEs with stellar masses lower than $10^{10.5} \mathrm{M}_{\odot}$ where we do not see a clear difference in the stellar mass 


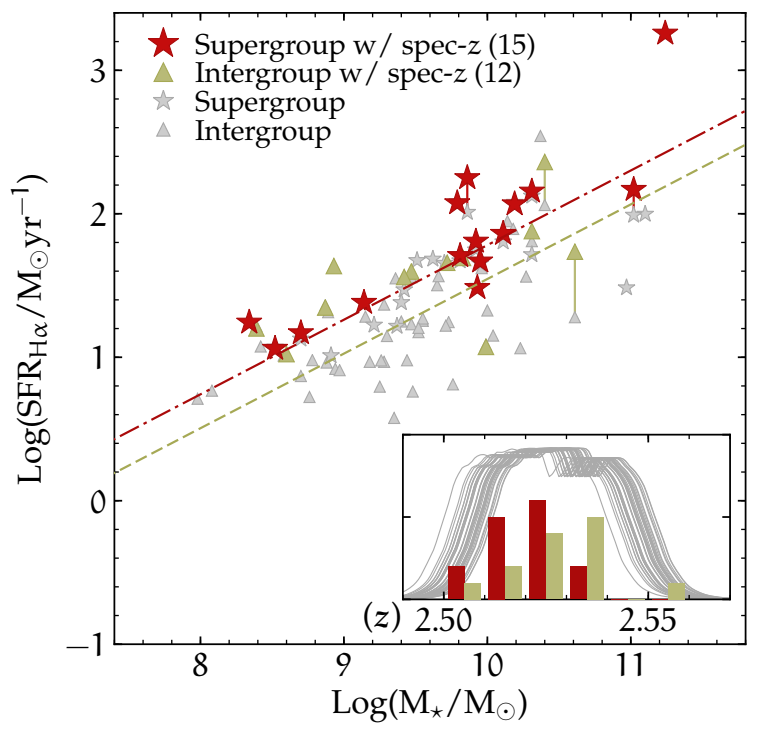

Figure C2. The same as Fig. 6, but we here highlight HAEs with spectroscopic confirmations by colours (reds: supergroup HAEs, yellows: intergroup HAEs). We correct filter flux losses of spec- $z$ sources where the spatial dependence on the filter centre wavelength is corrected. Grey points show the HAEs in each density without the correction of the filter flux loss. Variation of wavelength shift of the narrowband filter and redshift distributions of the HAE samples are shown in the inset right below. The red and yellow histograms show HAEs in the supergroup and the intergroup regions, respectively.

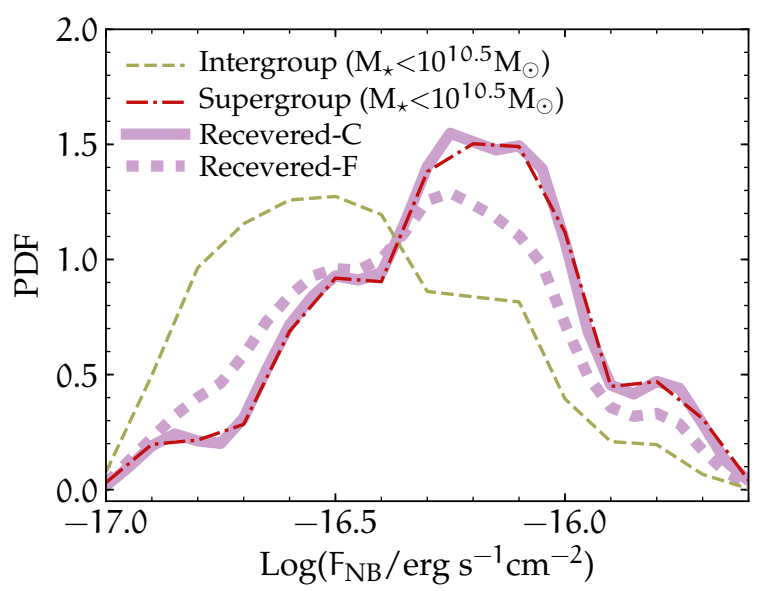

Figure C3. Probability density distributions (PDFs) of narrowband fluxes of HAEs in the supergroup (red dash-dot) and the intergroup (yellow dashed) regions. Purple solid and dotted curves are recovered PDFs by assuming the uniform redshift distribution (C) $z=2.51-2.535$ and (F) 2.49-2.56, respectively. We employ only HAEs at the stellar mass lower than $10^{10.5} \mathrm{M}_{\odot}$ to minimise the effects from the higher cutoff in the stellar mass function of the group HAEs.

distribution between the group HAEs and the intergroup HAEs. The assumption C $(z=2.51-2.535)$ well recovers the obtained narrowband distribution of HAEs in the supergroup region, indicating that the filter flux loss should be ignorable at this redshift range. On the other hand, we see a slight difference in the case of more homogeneous redshift distribution over the narrowband filter $(F)$. Since the case
F presumes that galaxies are homogeneously located at the wider redshift range even in which the filter flux loss is significant. The objects with significant flux losses are recovered as fainter sources, and then those narrowband flux distribution is slightly shifted towards the fainter side. However, the margin of flux distribution between supergroup and intergroup HAEs seems to remain consistent, suggesting that the only filter profile cannot explain the enhanced star-forming activities in the dense cores. However, these results also note that we cannot fully ignore this error as some sources show significant flux losses due to the non-top-hat function of the narrowband filter.

Lastly, one may wonder about the possibility that we are missing fainter HAEs in higher-densities. We stress that the limiting magnitudes of $N B 2315$ and $K_{S}$ bands have only variations of smaller than $0.1 \mathrm{mag}$ over the field of view, and we do not see a clear difference of sky background in source photometries of HAEs with the SExtractor as a function of the mean projected separation. Indeed, we reconstruct the object masks based on the combined science images by the one-stage process, and thus such errors from oversubtraction of sky background can be minimised. These suggest that spatial dependence of the sampling completeness should be ignorable. Thus, at least narrowband flux distribution in high densities should not be caused by observational bias. Furthermore, we cannot identify the prominent environmental dependence of the $\mathrm{H} \alpha$ luminosity function at the faint end. Considering all the various factors together, we conclude that enhanced star formation of HAEs in the supergroup regions is a more likely intrinsic trend rather than those resulting from any systematic error.

This paper has been typeset from a $\mathrm{T}_{\mathrm{E}} \mathrm{X} / \mathrm{LAT}_{\mathrm{E}} \mathrm{X}$ file prepared by the author. 